\title{
Astérisque
}

\section{Alain CONNES \\ Brisure de symétrie spontanée et géométrie du point de vue spectral}

Astérisque, tome 241 (1997), Séminaire Bourbaki, exp. $\mathrm{n}^{\circ} 816$, p. 313-349

<http://www.numdam.org/item?id=SB_1995-1996_38_313_0>

C Société mathématique de France, 1997, tous droits réservés.

L'accès aux archives de la collection « Astérisque » (http://smf4.emath.fr/ Publications/Asterisque/) implique l'accord avec les conditions générales d'utilisation (http://www.numdam.org/conditions). Toute utilisation commerciale ou impression systématique est constitutive d'une infraction pénale. Toute copie ou impression de ce fichier doit contenir la présente mention de copyright.

\section{Numdam}

Article numérisé dans le cadre du programme Numérisation de documents anciens mathématiques http://www.numdam.org/ 


\section{BRISURE DE SYMÉTRIE SPONTANÉE ET GÉOMÉTRIE DU POINT DE VUE SPECTRAL par Alain CONNES}

\section{GÉNÉRALITÉS}

La géométrie de Riemann admet pour données préalables une variété $M$ dont les points $x \in M$ sont localement paramétrés par un nombre fini de coordonnées réelles $x^{\mu}$, et la métrique donnée par l'élément de longueur infinitésimal

$$
d s^{2}=g_{\mu \nu} d x^{\mu} d x^{\nu}
$$

La distance entre deux points $x, y \in M$ est donnée par

$$
d(x, y)=\operatorname{Inf} \text { Longueur } \gamma
$$

où $\gamma$ varie parmi les arcs joignant $x$ à $y$, et

$$
\text { Longueur } \gamma=\int_{x}^{y} d s \text {. }
$$

La théorie de Riemann est à la fois assez souple pour fournir (au prix d'un changement de signe) un bon modèle de l'espace temps de la relativité générale et assez restrictive pour mériter le nom de géométrie. Le point essentiel est que le calcul différentiel et intégral permet de passer du local au global et que les notions simples de la géométrie euclidienne telle celle de droite continuent à garder un sens. L'équation des géodésiques :

$$
\frac{d^{2} x^{\mu}}{d t^{2}}=-\Gamma_{\nu \rho}^{\mu} \frac{d x^{\nu}}{d t} \frac{d x^{\rho}}{d t}
$$

(où $\Gamma_{\nu \rho}^{\mu}=\frac{1}{2} g^{\mu \alpha}\left(g_{\alpha \nu, \rho}+g_{\alpha \rho, \nu}-g_{\nu \rho, \alpha}\right)$ ) pour la métrique $d x^{2}+d y^{2}+d z^{2}-$ $(1-2 V(x, y, z)) d t^{2}$ donne l'équation de Newton dans le potentiel $V$ (cf. [W] pour un 
énoncé plus précis). Les données expérimentales récentes sur les pulsars binaires confirment [DT] la relativité générale et l'adéquation de la géométrie de Riemann comme modèle de l'espace temps à des échelles suffisamment grandes. La question $([R])$ de l'adéquation de cette géométrie comme modèle de l'espace temps à très courte échelle est controversée mais la longueur de Planck

$$
\ell_{p}=\left(G \hbar / c^{3}\right)^{1 / 2} \sim 10^{-33} \mathrm{~cm}
$$

est considérée comme la limite naturelle sur la détermination précise des coordonnées d'espace temps d'un évènement. (Voir par exemple $[\mathrm{F}]$ ou $[\mathrm{DFR}]$ pour l'argument physique, utilisant la mécanique quantique, qui établit cette limite.)

Dans cet exposé nous présentons une nouvelle notion d'espace géométrique qui en abandonnant le rôle central joué par les points de l'espace permet une plus grande liberté dans la description de l'espace temps à courte échelle. Le cadre proposé est suffisamment général pour traiter les espaces discrets, les espaces riemanniens, les espaces de configurations de la théorie quantique des champs et les duaux des groupes discrets non nécessairement commutatifs. Le problème principal est d'adapter à ce cadre général les notions essentielles de la géométrie et en particulier le calcul infinitésimal. Le formalisme opératoriel de la mécanique quantique joint à l'analyse des divergences logarithmiques de la trace des opérateurs donnent la généralisation cherchée du calcul différentiel et intégral (Section II). Nous donnons quelques applications directes de ce calcul (Théorèmes $1,2,4)$.

La donnée d'un espace géométrique est celle d'un triplet spectral :

$$
(\mathcal{A}, \mathcal{H}, D)
$$

où $\mathcal{A}$ est une algèbre involutive d'opérateurs dans l'espace de Hilbert $\mathcal{H}$ et $D$ un opérateur autoadjoint non borné dans $\mathcal{H}$. L'algèbre involutive $\mathcal{A}$ correspond à la donnée de l'espace $M$ selon la dualité Espace $\leftrightarrow$ Algèbre, classique en géométrie algébrique. L'opérateur $D^{-1}=d s$ correspond à l'élément de longueur infinitésimal de la géométrie de Riemann.

Il y a deux différences évidentes entre cette géométrie spectrale et la géométrie riemannienne. La première est que nous ne supposerons pas en général la commutativité de l'algèbre $\mathcal{A}$. La deuxième est que $d s$, étant un opérateur, ne commute pas avec les éléments de $\mathcal{A}$, même quand $\mathcal{A}$ est commutative.

Comme nous le verrons, des relations de commutation très simples entre $d s$ et l'algèbre $\mathcal{A}$, jointes à la dualité de Poincaré caractérisent les triplets spectraux (6) qui proviennent de variétés riemanniennes (Théorème 6). Quand l'algèbre $\mathcal{A}$ est commutative 
sa fermeture normique dans $\mathcal{H}$ est l'algèbre des fonctions continues sur un espace compact $M$. Un point de $M$ est un caractère de $\overline{\mathcal{A}}$, i.e. un homomorphisme de $\overline{\mathcal{A}}$ dans $\mathbb{C}$

$$
\begin{gathered}
\chi: \overline{\mathcal{A}} \rightarrow \mathbb{C}, \chi(a+b)=\chi(a)+\chi(b), \chi(\lambda a)=\lambda \chi(a), \chi(a b)=\chi(a) \chi(b), \\
\forall a, b \in \overline{\mathcal{A}}, \forall \lambda \in \mathbb{C} .
\end{gathered}
$$

Soit par exemple $\mathcal{A}$ l'algèbre $\mathbb{C} \Gamma$ d'un groupe discret $\Gamma$ agissant dans l'espace de Hilbert $\mathcal{H}=\ell^{2}(\Gamma)$ de la représentation régulière (gauche) de $\Gamma$. Quand le groupe $\Gamma$ et donc l'algèbre $\mathcal{A}$ sont commutatifs les caractères de $\overline{\mathcal{A}}$ sont les éléments du dual de Pontrjagin de $\Gamma$

$$
\hat{\Gamma}=\left\{\chi: \Gamma \rightarrow U(1) ; \chi\left(g_{1} g_{2}\right)=\chi\left(g_{1}\right) \chi\left(g_{2}\right) \quad \forall g_{1}, g_{2} \in \Gamma\right\}
$$

Les notions élémentaires de la géométrie différentielle pour l'espace $\hat{\Gamma}$ continuent à garder un sens dans le cas général où $\Gamma$ n'est plus commutatif grâce au dictionnaire suivant dont la colonne de droite n'utilise pas la commutativité de l'algèbre $\mathcal{A}$ :

Espace $X$

Fibré vectoriel

Forme différentielle de degré $k$

Homologie de de Rham

Algèbre $\mathcal{A}$

Module projectif de type fini Cycle de Hochschild de dimension $k$ Cocycle de Hochschild de dimension $k$ Cohomologie cyclique de $\mathcal{A}$

L'intérêt de la généralisation ci-dessus au cas non commutatif est illustré par exemple par la preuve de la conjecture de Novikov pour les groupes $\Gamma$ qui sont hyperboliques [CM1].

Dans le cas général la notion de point, donnée par (7), est de peu d'intérêt ; par contre celle de mesure de probabilité garde tout son sens. Une telle mesure $\varphi$ est une forme linéaire positive sur $\mathcal{A}$ telle que $\varphi(1)=1$

$$
\varphi: \overline{\mathcal{A}} \rightarrow \mathbb{C}, \varphi\left(a^{*} a\right) \geq 0, \quad \forall a \in \overline{\mathcal{A}}, \varphi(1)=1
$$

$\mathrm{Au}$ lieu de mesurer les distances entre les points de l'espace par la formule (2) nous mesurons les distances entre états $\varphi, \psi$ sur $\overline{\mathcal{A}}$ par une formule duale qui implique un sup au lieu d'un inf et n'utilise pas les arcs tracés dans l'espace

$$
d(\varphi, \psi)=\operatorname{Sup}\{|\varphi(a)-\psi(a)| ; a \in \mathcal{A},\|[D, a]\| \leq 1\} .
$$


Vérifions que cette formule redonne la distance géodésique dans le cas riemannien. Soit $M$ une variété riemannienne compacte munie d'une $K$-orientation, i.e. d'une structure spinorielle. Le triplet spectral $(\mathcal{A}, \mathcal{H}, D)$ associé est donné par la représentation

$$
(f \xi)(x)=f(x) \xi(x) \quad \forall x \in M, f \in \mathcal{A}, \xi \in \mathcal{H}
$$

de l'algèbre des fonctions sur $M$ dans l'espace de Hilbert

$$
\mathcal{H}=L^{2}(M, S)
$$

des sections de carré intégrable du fibré des spineurs.

L'opérateur $D$ est l'opérateur de Dirac (cf. [L-M]). On vérifie immédiatement que le commutateur $[D, f], f \in \mathcal{A}$ est l'opérateur de multiplication de Clifford par le gradient $\nabla f$ de $f$ et que sa norme hilbertienne est :

$$
\|[D, f]\|=\operatorname{Sup}_{x \in M}\|\nabla f\|=\text { Norme lipschitzienne de } f .
$$

Soient $x, y \in M$ et $\varphi, \psi$ les caractères correspondants : $\varphi(f)=f(x), \psi(f)=f(y)$. $\forall f \in \mathcal{A}$, la formule (10) donne le même résultat que la formule (2), i.e. donne la distance géodésique entre $x$ et $y$.

Contrairement à (2) la formule duale (10) garde un sens en général et en particulier pour les espaces discrets ou totalement discontinus.

La notion usuelle de dimension d'un espace est remplacée par un spectre de dimension qui est un sous-ensemble de $\mathbb{C}$ dont la partie réelle est bornée supérieurement par $\alpha>0$ si

$$
\lambda_{n}^{-1}=O\left(n^{-\alpha}\right)
$$

où $\lambda_{n}$ est la $n$-ième valeur propre de $|D|$.

La relation entre le local et le global est donnée par la formule locale de l'indice (Théorème 4) ([CM2]).

La propriété caractéristique des variétés différentiables qui est transposée au cas non commutatif est la dualité de Poincaré. La dualité de Poincaré en homologie ordinaire est insuffisante pour caractériser le type d'homotopie des variétés ([Mi-S]) mais les résultats de D. Sullivan ([S2]) montrent (dans le cas PL, simplement connexe, de dimension $\geq 5$ et en ignorant le nombre premier 2) qu'il suffit de remplacer l'homologie ordinaire par la $K O$-homologie. 
De plus la $K$-homologie admet grâce aux résultats de Brown Douglas Fillmore, Atiyah et Kasparov une traduction algébrique très simple, donnée par

Espace $X \quad$ Algèbre $\mathcal{A}$

$K_{1}(X) \quad$ Classe d'homotopie stable de triplet

spectral $(\mathcal{A}, \mathcal{H}, D)$

$K_{0}(X) \quad$ Classe d'homotopie stable de triplet

spectral $\mathbb{Z} / 2$ gradué

(i.e. pour $K_{0}$ on suppose que $\mathcal{H}$ est $\mathbb{Z} / 2$ gradué par $\gamma, \gamma=\gamma^{*}, \gamma^{2}=1$ et que $\gamma a=a \gamma \quad \forall a \in \mathcal{A}, \gamma D=-D \gamma)$.

Cette description suffit pour la $K$-homologie complexe qui est périodique de période 2 . Dans le cas non commutatif la classe fondamentale d'un espace est une classe $\mu$ de $K R$-homologie pour l'algèbre $\mathcal{A} \otimes \mathcal{A}^{0}$ munie de l'involution

$$
\tau\left(x \otimes y^{0}\right)=y^{*} \otimes\left(x^{*}\right)^{0} \quad \forall x, y \in \mathcal{A}
$$

où $\mathcal{A}^{0}$ désigne l'algèbre opposée de $\mathcal{A}$. Le produit intersection de Kasparov $[\mathrm{K}]$ permet de formuler la dualité de Poincaré par l'invertibilité de $\mu$. La $K R$-homologie est périodique de période 8 et la dimension modulo 8 est spécifiée par les règles de commutation suivantes, où $J$ est une isométrie antilinéaire dans $\mathcal{H}$ qui implémente l'involution $\tau$

$$
J x J^{-1}=\tau(x) \quad \forall x \in \mathcal{A} \otimes \mathcal{A}^{0}
$$

On a $J^{2}=\varepsilon, J D=\varepsilon^{\prime} D J, J \gamma=\varepsilon^{\prime \prime} \gamma J$ où $\varepsilon, \varepsilon^{\prime}, \varepsilon^{\prime \prime} \in\{-1,1\}$ et si $n$ désigne la dimension modulo 8

$$
\begin{array}{lllrrrrrr}
n= & 0 & 1 & 2 & 3 & 4 & 5 & 6 & 7 \\
\varepsilon & 1 & 1 & -1 & -1 & -1 & -1 & 1 & 1 \\
\varepsilon^{\prime} & 1 & -1 & 1 & 1 & 1 & -1 & 1 & 1 \\
\varepsilon^{\prime \prime} & 1 & & -1 & & 1 & & -1 &
\end{array}
$$

L'isométrie antilinéaire $J$ est donnée dans le cas riemannien par l'opérateur de conjugaison de charge et dans le cas non commutatif par l'opérateur de Tomita [Ta] qui, 


\section{A. CONNES}

dans le cas où une algèbre d'opérateurs $\mathcal{A}$ admet un vecteur cyclique qui est cyclique pour le commutant $\mathcal{A}^{\prime}$, établit un antiisomorphisme

$$
a \in \mathcal{A}^{\prime \prime} \rightarrow J a^{*} J^{-1} \in \mathcal{A}^{\prime}
$$

La donnée de $\mu$ ne spécifie que la classe d'homotopie stable du triplet spectral $(\mathcal{A}, \mathcal{H}, D)$ muni de l'isométrie $J$ (et de la $\mathbb{Z} / 2$ graduation $\gamma$ si $n$ est pair). La non trivialité de cette classe d'homotopie est visible dans la forme d'intersection

$$
K(\mathcal{A}) \times K(\mathcal{A}) \rightarrow \mathbb{Z}
$$

donnée par l'indice de Fredholm de $D$ à coefficient dans $K\left(\mathcal{A} \otimes \mathcal{A}^{0}\right)$.

Pour comparer les triplets spectraux dans la classe $\mu$, nous utiliserons la fonctionnelle spectrale suivante

$$
\operatorname{Trace}(\varpi(D))
$$

où $\varpi: \mathbb{R} \rightarrow \mathbb{R}_{+}$est une fonction positive convenable.

L'algèbre $\mathcal{A}$ une fois fixée, une géométrie spectrale est déterminée par la classe d'équivalence unitaire du triplet spectral $(\mathcal{A}, \mathcal{H}, D)$ avec l'isométrie $J$. Si l'on note $\pi$ la représentation de $\mathcal{A}$ dans $\mathcal{H}$, l'équivalence unitaire entre $\left(\pi_{1}, \mathcal{H}_{1}, D_{1}, J_{1}\right)$ et $\left(\pi_{2}, \mathcal{H}_{2}, D_{2}, J_{2}\right)$ signifie qu'il existe un unitaire $U: \mathcal{H}_{1} \rightarrow \mathcal{H}_{2}$ tel que

$$
U \pi_{1}(a) U^{*}=\pi_{2}(a) \quad \forall a \in \mathcal{A}, U D_{1} U^{*}=D_{2}, U J_{1} U^{*}=J_{2}
$$

(et $U \gamma_{1} U^{*}=\gamma_{2}$ dans le cas où $n$ est pair).

Le groupe $\operatorname{Aut}(\mathcal{A})$ des automorphismes de l'algèbre involutive $\mathcal{A}$ agit sur l'ensemble des géométries spectrales par composition

$$
\pi^{\prime}(a)=\pi\left(\alpha^{-1}(a)\right) \quad \forall a \in \mathcal{A}, \alpha \in \operatorname{Aut}(\mathcal{A})
$$

Le sous-groupe $\operatorname{Aut}^{+}(\mathcal{A})$ des automorphismes qui préservent la classe $\mu$ agit sur la classe d'homotopie stable déterminée par $\mu$ et préserve par construction la fonctionnelle d'action (19). En général ce groupe est non compact, et il coïncide par exemple dans le cas riemannien avec le groupe $\operatorname{Diff}^{+}(M)$ des difféomorphismes qui préservent la $K$-orientation, i.e. la structure spinorielle de $M$. A l'inverse le groupe d'isotropie d'une géométrie donnée, est automatiquement compact (pour $\mathcal{A}$ unifère). Ceci montre que la fonctionnelle d'action (19) donne automatiquement naissance au phénomène de brisure de symétrie spontanée (Figure 1). 
Nous montrerons que pour un choix convenable de l'algèbre $\mathcal{A}$ la fonctionnelle d'action (19) ajoutée au terme $\langle\xi, D \xi\rangle, \xi \in \mathcal{H}$ donne le modèle standard de Glashow-WeinbergSalam couplé à la gravitation. L'algèbre $\mathcal{A}$ est le produit tensoriel de l'algèbre des fonctions sur un espace riemannien $M$ par une algèbre non commutative de dimension finie dont les données phénoménologiques spécifient la géométrie spectrale.

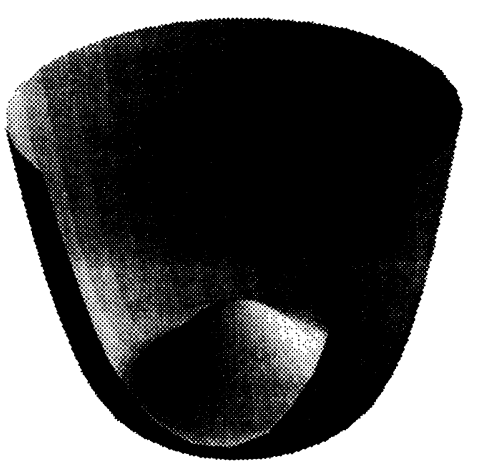

Figure 1

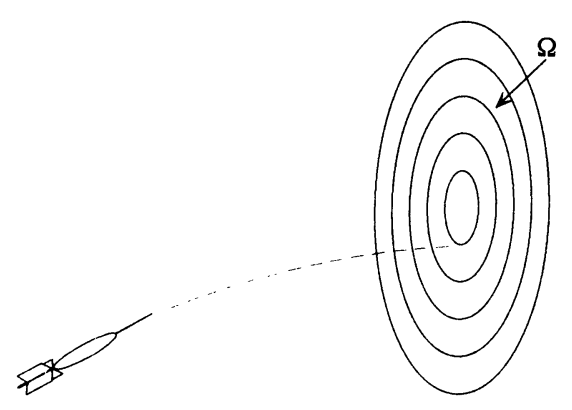

Figure 2 


\section{UN CALCUL INFINITÉSIMAL}

Nous montrons comment le formalisme opératoriel de la mécanique quantique permet de donner un sens précis à la notion de variable infinitésimale. La notion d'infinitésimal est sensée avoir un sens intuitif évident. Elle résiste cependant fort bien aux essais de formalisation donnés par exemple par l'analyse non standard. Ainsi, pour prendre un exemple précis ([B-W]), soit $d p(x)$ la probabilité pour qu'une fléchette lancée au hasard sur la cible $\Omega$ termine sa course au point $x \in \Omega$ (Figure 2). Il est clair que $d p(x)<\varepsilon \quad \forall \varepsilon>0$ et que néanmoins la réponse $d p(x)=0$ n'est pas satisfaisante. Le formalisme usuel de la théorie de la mesure ou des formes différentielles contourne le problème en donnant un sens à l'expression

$$
\int f(x) d p(x) \quad f: \Omega \rightarrow \mathbb{C}
$$

mais est insuffisant pour donner un sens par exemple à $e^{-\frac{1}{d p(x)}}$. La réponse, à savoir un réel non standard, fournie par l'analyse non standard, est également décevante : tout réel non standard détermine canoniquement un sous-ensemble non Lebesgue mesurable de l'intervalle $[0,1]$ de sorte qu'il est impossible ([Ste]) d'en exhiber un seul. Le formalisme que nous proposons donnera une réponse substantielle et calculable à cette question.

Le cadre est fixé par un espace de Hilbert séparable $\mathcal{H}$ décomposé comme somme directe de deux sous-espaces de dimension infinie. Donner cette décomposition revient à donner l'opérateur linéaire $F$ dans $\mathcal{H}$ qui est l'identité, $F \xi=\xi$, sur le premier sousespace et moins l'identité, $F \xi=-\xi$ sur le second ; on a

$$
F=F^{*}, F^{2}=1
$$

Le cadre ainsi déterminé est unique à isomorphisme près. Le début du dictionnaire qui traduit les notions classiques en language opératoriel est le suivant : 
Classique

Variable complexe

Variable réelle

Infinitésimal

Infinitésimal d'ordre $\alpha$

Différentielle d'une variable réelle ou complexe

Intégrale d'un infinitésimal d'ordre 1
Quantique

Opérateur dans $\mathcal{H}$

Opérateur autoadjoint

Opérateur compact

Opérateur compact dont les valeurs caractéristiques $\mu_{n}$ vérifient $\mu_{n}=O\left(n^{-\alpha}\right), n \rightarrow \infty$

$$
d f=[F, f]=F f-f F
$$

$f T=$ Coefficient de la divergence

logarithmique dans la trace de $T$.

Les deux premières lignes du dictionnaire sont familières en mécanique quantique. L'ensemble des valeurs d'une variable complexe correspond au spectre d'un opérateur. Le calcul fonctionnel holomorphe donne un sens à $f(T)$ pour toute fonction holomorphe $f$ sur le spectre de $T$. Les fonctions holomorphes sont les seules à opérer dans cette généralité ce qui reflète la différence entre l'analyse complexe et l'analyse réelle où les fonctions boréliennes arbitraires opèrent. Quand $T=T^{*}$ est autoadjoint $f(T)$ a un sens pour toute fonction borélienne $f$. Notons que toute variable aléatoire usuelle $X$ sur un espace de probabilité, $(\Omega, P)$ peut être trivialement considérée comme un opérateur autoadjoint. On prend $\mathcal{H}=L^{2}(\Omega, P)$ et

$$
(T \xi)(p)=X(p) \xi(p) \quad \forall p \in \Omega, \xi \in \mathcal{H}
$$

La mesure spectrale de $T$ redonne la probabilité $P$.

Passons à la troisième ligne du dictionnaire. Nous cherchons des "variables infinitésimales", i.e. des opérateurs $T$ dans $\mathcal{H}$ tels que

$$
\|T\|<\varepsilon \quad \forall \varepsilon>0
$$

où $\|T\|=\operatorname{Sup}\{\|T \xi\| ;\|\xi\|=1\}$ est la norme d'opérateur. Bien entendu si l'on prend (4) au pied de la lettre on obtient $\|T\|=0$ et $T=0$ est la seule solution. Mais on peut affaiblir (4) de la manière suivante

$$
\forall \varepsilon>0, \exists \text { sous-espace de dimension finie } E \subset \mathcal{H} \text { tel que }\left\|T / E^{\perp}\right\|<\varepsilon
$$


où $E^{\perp}$ désigne l'orthogonal de $E$ dans $\mathcal{H}$

$$
E^{\perp}=\{\xi \in \mathcal{H} ;\langle\xi, \eta\rangle=0 \quad \forall \eta \in E\}
$$

qui est un sous-espace de codimension finie de $\mathcal{H}$. Le symbole $T / E^{\perp}$ désigne la restriction de $T$ à ce sous-espace

$$
T / E^{\perp}: E^{\perp} \rightarrow \mathcal{H}
$$

Les opérateurs qui satisfont la condition (5) sont les opérateurs compacts, i.e. sont caractérisés par la compacité normique de l'image de la boule unité de $\mathcal{H}$. L'opérateur $T$ est compact ssi $|T|=\sqrt{T^{*} T}$ est compact, et ceci a lieu ssi le spectre de $|T|$ est une suite de valeurs propres $\mu_{0} \geq \mu_{1} \geq \mu_{2} \ldots, \mu_{n} \downarrow 0$.

Ces valeurs propres sont les valeurs caractéristiques de $T$ et on a

$$
\mu_{n}(T)=\operatorname{Inf}\{\|T-R\| ; R \text { opérateur de rang } \leq n\}
$$

$$
\mu_{n}(T)=\operatorname{Inf}\left\{\left\|T / E^{\perp}\right\| ; \operatorname{dim} E \leq n\right\}
$$

Les opérateurs compacts forment un idéal bilatère $\mathcal{K}$ dans l'algèbre $\mathcal{L}(\mathcal{H})$ des opérateurs bornés dans $\mathcal{H}$ de sorte que les règles algébriques élémentaires du calcul infinitésimal sont vérifiées.

La taille d'un infinitésimal $T \in \mathcal{K}$ est gouvernée par l'ordre de décroissance de la suite $\mu_{n}=\mu_{n}(T)$, quand $n \rightarrow \infty$. En particulier pour tout réel positif $\alpha$ la condition

$$
\mu_{n}(T)=O\left(n^{-\alpha}\right) \quad \text { quand } n \rightarrow \infty
$$

(i.e. il existe $C>0$ tel que $\mu_{n}(T) \leq C n^{-\alpha} \quad \forall n \geq 1$ ) définit les infinitésimaux d'ordre $\alpha$. Ils forment de même un idéal bilatère, comme on le voit en utilisant (8), (cf. [Co]) et de plus

$$
T_{j} \text { d'ordre } \alpha_{j} \Rightarrow T_{1} T_{2} \text { d'ordre } \alpha_{1}+\alpha_{2} \text {. }
$$

(Pour $\alpha<1$ l'idéal correspondant est un idéal normé obtenu par interpolation réelle entre l'idéal $\mathcal{L}^{1}$ des opérateurs traçables et l'idéal $\mathcal{K}([\mathrm{Co}])$.) Ainsi, hormis la commutativité, les propriétés intuitives du calcul infinitésimal sont vérifiées.

Comme la taille d'un infinitésimal est mesurée par une suite $\mu_{n} \rightarrow 0$ il pourrait sembler inutile d'utiliser le formalisme opératoriel. Il suffirait de remplacer l'idéal $\mathcal{K}$ 
de $\mathcal{L}(\mathcal{H})$ par l'idéal $c_{0}(\mathbb{N})$ des suites convergeant vers 0 dans l'algèbre $\ell^{\infty}(\mathbb{N})$ des suites bornées. Cette version commutative ne convient pas car tout élément de $\ell^{\infty}(\mathbb{N})$ a un spectre ponctuel et une mesure spectrale discrète. Ce n'est que la non commutativité de $\mathcal{L}(\mathcal{H})$ qui permet la coexistence de variables ayant un spectre de Lebesgue avec des variables infinitésimales.

En fait la ligne suivante du dictionnaire utilise de manière cruciale la non commutativité de $\mathcal{L}(\mathcal{H})$. La différentielle $d f$ d'une variable réelle ou complexe

$$
d f=\Sigma \frac{\partial f}{\partial x^{\mu}} d x^{\mu}
$$

est remplacée par le commutateur

$$
d f=[F, f]
$$

Le passage de (11) à (12) est semblable à la transition du crochet de Poisson $\{f, g\}$ de deux observables $f, g$ de la mécanique classique, au commutateur $[f, g]=f g-g f$ d'observables quantiques.

Etant donnée une algèbre $\mathcal{A}$ d'opérateurs dans $\mathcal{H}$, la dimension de l'espace correspondant (au sens du dictionnaire 1) est gouvernée par la taille des différentielles $d f$, $f \in \mathcal{A}$. En dimension $p$ on a

$$
\text { đf d'ordre } \frac{1}{p}, \forall f \in \mathcal{A} .
$$

Nous verrons très vite des exemples concrets où $p$ est la dimension de Hausdorff d'un ensemble de Julia. Des manipulations algébriques très simples sur la fonctionnelle

$$
\tau\left(f^{0}, \ldots, f^{n}\right)=\operatorname{Trace}\left(f^{0} d f^{1} \ldots d f^{n}\right) \quad n \text { impair, } n>p
$$

montrent que $\tau$ est un cocycle cyclique et permettent de transposer les idées de la topologie différentielle en exploitant l'intégralité du cocycle $\tau$, i.e. $\left\langle\tau, K_{1}(\mathcal{A})\right\rangle \subset \mathbb{Z}$.

Si le dictionnaire s'arrêtait là, il nous manquerait un outil vital du calcul infinitésimal, la localité, i.e. la possibilité de négliger les infinitésimaux d'ordre $>1$ dans un calcul. Dans notre cadre les infinitésimaux d'ordre $>1$ sont contenus dans l'idéal bilatère suivant

$$
\left\{T \in \mathcal{K} ; \mu_{n}(T)=o\left(\frac{1}{n}\right)\right\}
$$

où le petit $o$ à la signification usuelle. 


\section{A. CONNES}

Ainsi, si nous utilisons la trace comme dans (16) pour intégrer, nous rencontrons deux problèmes :

a) les infinitésimaux d'ordre 1 ne sont pas dans le domaine de la trace,

b) la trace des infinitésimaux d'ordre $>1$ n'est pas nulle.

Le domaine naturel de la trace est l'idéal bilatère $\mathcal{L}^{1}(\mathcal{H})$ des opérateurs traçables

$$
\mathcal{L}^{1}=\left\{T \in \mathcal{K} ; \sum_{o}^{\infty} \mu_{n}(T)<\infty\right\} .
$$

La trace d'un opérateur $T \in \mathcal{L}^{1}(\mathcal{H})$ est donnée par la somme

$$
\operatorname{Trace}(T)=\sum\left\langle T \xi_{i}, \xi_{i}\right\rangle
$$

indépendamment du choix de la base orthonormale $\left(\xi_{i}\right)$ de $\mathcal{H}$. On a

$$
\operatorname{Trace}(T)=\sum_{o}^{\infty} \mu_{n}(T) \quad \forall T \geq 0 .
$$

Soit $T \geq 0$ un infinitésimal d'ordre 1 , le seul contrôle sur $\mu_{n}(T)$ est

$$
\mu_{n}(T)=O\left(\frac{1}{n}\right)
$$

ce qui ne suffit pas pour assurer la finitude de (20). Ceci précise la nature du problème a) et de même pour b) puisque la trace ne s'annule pas pour le plus petit idéal de $\mathcal{L}(\mathcal{H})$, l'idéal $\mathcal{R}$ des opérateurs de rang fini.

Ces deux problèmes sont résolus par la trace de Dixmier [Dx], i.e. par l'analyse suivante de la divergence logarithmique des traces partielles

$$
\operatorname{Trace}_{N}(T)=\sum_{o}^{N-1} \mu_{n}(T), T \geq 0 .
$$

Il est utile de définir $\operatorname{Trace}_{\Lambda}(T)$ pour tout $\Lambda>0$ par la formule d'interpolation

$$
\operatorname{Trace}_{\Lambda}(T)=\operatorname{Inf}\left\{\|A\|_{1}+\Lambda\|B\| ; A+B=T\right\}
$$

où $\|A\|_{1}$ est la norme $\mathcal{L}^{1}$ de $A,\|A\|_{1}=$ Trace $|A|$. Cette formule coïncide avec (22) pour $\Lambda$ entier et donne l'interpolation affine par morceaux. On a de plus ([Co])

$$
\operatorname{Trace}_{\Lambda}\left(T_{1}+T_{2}\right) \leq \operatorname{Trace}_{\Lambda}\left(T_{1}\right)+\operatorname{Trace}_{\Lambda}\left(T_{2}\right) \quad \forall \Lambda
$$




$$
\operatorname{Trace}_{\Lambda_{1}+\Lambda_{2}}\left(T_{1}+T_{2}\right) \geq \operatorname{Trace}_{\Lambda_{1}}\left(T_{1}\right)+\operatorname{Trace}_{\Lambda_{2}}\left(T_{2}\right) \quad \forall \Lambda_{1}, \Lambda_{2}
$$

où $T_{1}, T_{2}$ sont positifs pour (25).

Soit $T>0$ infinitésimal d'ordre 1 ; on a alors

$$
\operatorname{Trace}_{\Lambda}(T) \leq C \log \Lambda
$$

et la propriété remarquable d'additivité asymptotique du coefficient de la divergence logarithmique (26) est la suivante: $\left(T_{j} \geq 0\right)$,

$$
\left|\tau_{\Lambda}\left(T_{1}+T_{2}\right)-\tau_{\Lambda}\left(T_{1}\right)-\tau_{\Lambda}\left(T_{2}\right)\right| \leq 3 C \frac{\log (\log \Lambda)}{\log \Lambda}
$$

où, pour tout $T \geq 0$, on pose

$$
\tau_{\Lambda}(T)=\frac{1}{\log \Lambda} \int_{e}^{\Lambda} \frac{\operatorname{Trace}_{\mu}(T)}{\log \mu} \frac{d \mu}{\mu}
$$

qui est la moyenne de Cesaro sur le groupe $\mathbb{R}_{+}^{*}$ des échelles, de la fonction $\frac{\operatorname{Trace} \mu(T)}{\log \mu}$. Il résulte facilement de (27) que toute limite simple $\tau$ des fonctionnelles non linéaires $\tau_{\Lambda}$ définit une trace positive et linéaire sur l'idéal bilatère des infinitésimaux d'ordre 1

$$
\begin{array}{cc}
\tau\left(\lambda_{1} T_{1}+\lambda_{2} T_{2}\right)=\lambda_{1} \tau\left(T_{1}\right)+\lambda_{2} \tau\left(T_{2}\right) \quad \forall \lambda_{j} \in \mathbb{C} \\
\tau(T) \geq 0 \quad \forall T \geq 0 \\
\tau(S T)=\tau(T S) \quad \forall S \text { borné } \\
\tau(T)=0 \text { si } \mu_{n}(T)=o\left(\frac{1}{n}\right) .
\end{array}
$$

En pratique le choix du point limite $\tau$ est sans importance car dans tous les exemples importants (et en particulier comme corollaire des axiomes dans le cadre général, cf. Section IV) la condition suivante de mesurabilité est satisfaite :

$$
\tau_{\Lambda}(T) \text { est convergent quand } \Lambda \rightarrow \infty
$$

Pour les opérateurs mesurables la valeur de $\tau(T)$ est indépendante de $\tau$ et est notée

$$
f T \text {. }
$$

Le premier exemple intéressant est celui des opérateurs pseudodifférentiels $T$ sur une variété différentiable $M$. Quand $T$ est d'ordre 1 (au sens de (21)) il est mesurable et 
$f T$ est le résidu non commutatif de $T$ ([Wo], [Ka]). Ce résidu a une expression locale très simple en terme du noyau distribution $k(x, y), x, y \in M$. Quand $T$ est d'ordre 1 (au sens de (21)) le noyau $k(x, y)$ admet une divergence logarithmique au voisinage de la diagonale

$$
k(x, y)=a(x) \log |x-y|+0(1)
$$

où $|x-y|$ est la distance riemannienne dont le choix n'affecte pas la 1-densité $a(x)$. On a alors (à normalisation près)

$$
f T=\int_{M} a(x)
$$

et le terme de droite de cette formule se prolonge de manière quasi évidente à tous les opérateurs pseudodifférentiels (cf. [Wo]) si l'on note que le noyau d'un tel opérateur admet un développement asymptotique de la forme

$$
k(x, y)=\sum a_{k}(x, x-y)+a(x) \log |x-y|+0(1)
$$

où $a_{k}(x, \xi)$ est homogène de degré $-k$ en la variable $\xi$, et où la 1-densité $a(x)$ est définie de manière intrinsèque.

En fait le même principe de prolongement de $f$ à des infinitésimaux d'ordre $<1$ s'applique aux opérateurs hypoelliptiques et plus généralement (cf. Théorème 4) aux triplets spectraux dont le spectre de dimension est simple.

Après cette description, passons à des exemples. La variable infinitésimale $d p(x)$ qui donne la probabilité dans le jeu de fléchettes (Figure 2) est donnée par l'opérateur

$$
d p=\Delta^{-1}
$$

où $\Delta$ est le Laplacien de Dirichlet pour le domaine $\Omega$. Il agit dans l'espace de Hilbert $L^{2}(\Omega)$ ainsi que l'algèbre des fonctions $f\left(x_{1}, x_{2}\right), f: \Omega \rightarrow \mathbb{C}$, qui agissent par opérateurs de multiplication (cf. (3)). Le théorème de H. Weyl montre immédiatement que $d p$ est d'ordre 1 , que $f d p$ est mesurable et que

$$
f f d p=\int_{\Omega} f\left(x_{1}, x_{2}\right) d x_{1} \wedge d x_{2}
$$

donne la probabilité usuelle.

Montrons maintenant comment utiliser notre calcul infinitésimal pour donner un sens à des expressions telles que l'aire d'une variété de dimension 4, qui sont dépourvues de sens dans le calcul usuel. 
Il y a, à équivalence unitaire et multiplicité près, une seule quantification du calcul infinitésimal sur $\mathbb{R}$ qui soit invariante par translations et dilatations. Elle est donnée par la représentation de l'algèbre des fonctions $f$ sur $\mathbb{R}$ comme opérateurs de multiplication dans $L^{2}(\mathbb{R})$ (cf. (3)), alors que l'opérateur $F$ dans $\mathcal{H}=L^{2}(\mathbb{R})$ est la transformation de Hilbert ([St])

$$
(f \xi)(s)=f(s) \xi(s) \quad \forall s \in \mathbb{R}, \xi \in L^{2}(\mathbb{R}),(F \xi)(t)=\frac{1}{\pi i} \int \frac{\xi(s)}{s-t} d s .
$$

On a une description unitairement équivalente pour $S^{1}=P_{1}(\mathbb{R})$ avec $\mathcal{H}=L^{2}\left(S^{1}\right)$ et

$$
F e_{n}=\operatorname{Sign}(n) e_{n}, e_{n}(\tau)=\exp (i n \tau) \quad \forall \tau \in S^{1},(\operatorname{Sign}(0)=1)
$$

L'opérateur $d f=[F, f]$, pour $f \in L^{\infty}(\mathbb{R})$, est représenté par le noyau $\frac{1}{\pi i} k(s, t)$, avec

$$
k(s, t)=\frac{f(s)-f(t)}{s-t} .
$$

Comme $f$ et $F$ sont des opérateurs bornés, il en est de même de $d f=[F, f]$ pour toute $f$ mesurable bornée sur $S^{1}$, ce qui donne un sens à $|đ f|^{p}$ pour tout $p>0$. Soient par exemple $c \in \mathbb{C}$ et $J$ l'ensemble de Julia associé à l'itération de la transformation

$$
\varphi(z)=z^{2}+c, J=\partial B, B=\left\{z \in \mathbb{C} ; \operatorname{Sup}_{n \in \mathbb{N}}\left|\varphi^{n}(z)\right|<\infty\right\} .
$$

Pour $c$ petit $J$ est une courbe de Jordan et $B$ la composante bornée de son complément. Soit $Z: S^{1} \rightarrow J$ la restriction à $S^{1}=\partial D, D=\{z \in \mathbb{C},|z|<1\}$ d'une équivalence conforme $D \sim B$. Comme (par un résultat de D. Sullivan) la dimension de Hausdorff $p$ de $J$ est $>1$ (pour $c \neq 0$ ) la fonction $Z$ n'est nulle part à variation bornée et la valeur absolue $\left|Z^{\prime}\right|$ de la dérivée de $Z$ au sens des distributions n'a pas de sens. Cependant $|\Varangle Z|$ est bien défini et on a :

THÉORÈME 1.- a) $|\Varangle Z|$ est un infinitésimal d'ordre $\frac{1}{p}$.

b) Pour toute fonction continue $h$ sur $J$, l'opérateur $h(Z)|d Z|^{p}$ est mesurable.

c) $\exists \lambda>0$ :

$$
f h(Z)|d Z|^{p}=\lambda \int h d \Lambda_{p} \quad \forall h \in C(J),
$$

où $d \Lambda_{p}$ désigne la mesure de Hausdorff sur $J$.

L'énoncé a) utilise un résultat de V.V. Peller qui caractérise les fonctions $f$ pour lesquelles Trace $\left(|d f|^{\alpha}\right)<\infty$. La constante $\lambda$ gouverne le développement asymptotique de la distance dans $L^{\infty}\left(S^{1}\right)$ entre $Z$ et les fonctions rationnelles ayant au plus 
$n$-pôles hors du disque unité. Cette constante est de l'ordre de $\sqrt{p-1}$ et s'annule pour $p=1$. Cela tient à une propriété spécifique de la dimension 1 , à savoir que pour $f \in C^{\infty}\left(S^{1}\right) đ f$ n'est pas seulement d'ordre $\left(\operatorname{dim} S^{1}\right)^{-1}=1$ mais est traçable, avec

$$
\operatorname{Trace}\left(f^{0} d f^{1}\right)=\frac{1}{\pi i} \int_{S_{1}} f^{0} d f^{1} \quad \forall f^{0}, f^{1} \in C^{\infty}\left(S^{1}\right) .
$$

En fait par un résultat classique de Kronecker $₫ f$ est de rang fini ssi $f$ est une fraction rationnelle (cf. $[\mathrm{P}])$.

Le calcul différentiel quantique s'applique de la même manière à l'espace projectif $P_{1}(K)$ sur un corps local arbitraire $K$ (i.e. un corps localement compact non discret) et est invariant par le groupe des transformations projectives. Les cas spéciaux $K=\mathbb{C}$ et $K=\mathbb{H}$ (corps des quaternions) sont des cas particuliers du calcul sur les variétés compactes conformes orientées de dimension paire, $M=M_{2 n}$, qui se définit ainsi :

$$
\mathcal{H}=L^{2}\left(M, \Lambda^{n} T^{*}\right),(f \xi)(p)=f(p) \xi(p) \quad \forall f \in L^{\infty}(M), F=2 P-1
$$

où le produit scalaire sur l'espace de Hilbert des formes différentielles de degré $n=$ $\frac{1}{2} \operatorname{dim} M$ est donné par $\left\langle\omega_{1}, \omega_{2}\right\rangle=\int \omega_{1} \wedge * \omega_{2}$ et ne dépend que de la structure conforme de $M$. L'opérateur $P$ est le projecteur orthogonal sur le sous-espace des formes exactes.

Prenons d'abord $n=1$. Un calcul immédiat donne

$$
f d f d g=-\frac{1}{\pi} \int d f \wedge * d g \quad \forall f, g \in C^{\infty}(M) .
$$

Soit alors $X$ une application $\left(C^{\infty}\right)$ de $M$ dans l'espace $\mathbb{R}^{N}$ muni de la métrique riemannienne $g_{\mu \nu} d x^{\mu} d x^{\nu}$; on a

$$
f g_{\mu \nu} d X^{\mu} d X^{\nu}=-\frac{1}{\pi} \int_{M} g_{\mu \nu} d X^{\mu} \wedge * d X^{\nu}
$$

où le terme de droite est l'action de Polyakov de la théorie des cordes. Pour $n=4$ l'égalité (44) n'a pas lieu, l'action définie par le terme de droite n'est pas intéressante car elle n'est pas invariante conforme. Le terme de gauche est parfaitement défini par le calcul quantique et est invariant conforme : on a

THÉORÈME 2.- Soit $X$ une application $C^{\infty}$ de $M_{4}$ dans $\left(\mathbb{R}^{N}, g_{\mu \nu} d x^{\mu} d x^{\nu}\right)$,

$$
\begin{gathered}
f g_{\mu \nu}(X) d X^{\mu} d X^{\nu}=\left(16 \pi^{2}\right)^{-1} \int_{M} g_{\mu \nu}(X) \\
\left\{\frac{1}{3} r\left\langle d X^{\mu}, d X^{\nu}\right\rangle-\Delta\left\langle d X^{\mu}, d X^{\nu}\right\rangle+\left\langle\nabla d X^{\mu}, \nabla d X^{\nu}\right\rangle-\frac{1}{2}\left(\Delta X^{\mu}\right)\left(\Delta X^{\nu}\right)\right\} d v
\end{gathered}
$$


où, pour écrire le terme de droite, on utilise sur $M$ une structure riemannienne $\eta$ quelconque compatible avec la structure conforme. Ainsi la courbure scalaire $r$, le Laplacien $\Delta$ et la connection de Levi Civita $\nabla$ se réfèrent à $\eta$, mais le résultat n'en dépend pas.

Le théorème 2 est à rapprocher de la formule suivante qui exprime l'action de Hilbert Einstein comme l'aire d'une variété de dimension 4 (cf. [Kas] [K-W])

$$
f d s^{2}=\frac{-1}{96 \pi^{2}} \int_{M_{4}} r \sqrt{g} d^{4} x
$$

( $d v=\sqrt{g} d^{4} x$ est la forme volume et $d s=D^{-1}$ est l'élément de longueur, i.e. l'inverse de l'opérateur de Dirac).

Quand la métrique $g_{\mu \nu} d x^{\mu} d x^{\nu}$ sur $\mathbb{R}^{N}$ est invariante par translations, la fonctionnelle d'action du théorème 2 est donnée par l'opérateur de Paneitz sur $M$. C'est un opérateur d'ordre 4 qui joue le rôle du Laplacien en géométrie conforme ([B-O]). Son anomalie conforme a été calculée par T. Branson [B].

Reprenons le cas $n=2$ et modifions la structure conforme de $M$ par une différentielle de Beltrami $\mu(z, \bar{z}) d \bar{z} / d z,|\mu(z, \bar{z})|<1$ en utilisant pour définir les angles en $z \in M$

$$
X \in T_{z}(M) \rightarrow\langle X, d z+\mu(z, \bar{z}) d \bar{z}\rangle \in \mathbb{C}
$$

au lieu de $\langle X, d z\rangle$. Le calcul quantique sur $M$ associé à la nouvelle structure conforme s'obtient simplement en remplaçant l'opérateur $F$ par l'opérateur $F^{\prime}$,

$$
F^{\prime}=(\alpha F+\beta)(\beta F+\alpha)^{-1}, \alpha=\left(1-m^{2}\right)^{-1 / 2}, \beta=m\left(1-m^{2}\right)^{-1 / 2}
$$

où $m$ est l'opérateur dans $\mathcal{H}=L^{2}\left(M, \Lambda^{1} T^{*}\right)$ donné par l'endomorphisme du fibré vectoriel $\Lambda^{1} T^{*}=\Lambda^{(1,0)} \oplus \Lambda^{(0,1)}$ de matrice,

$$
m(z, \bar{z})=\left[\begin{array}{cc}
0 & \bar{\mu}(z, \bar{z}) d \bar{z} / d z \\
\mu(z, \bar{z}) d z / d \bar{z} & 0
\end{array}\right] .
$$

Les propriétés cruciales de l'opérateur $m \in \mathcal{L}(\mathcal{H})$ sont

$$
\|m\|<1, m=m^{*}, m f=f m \quad \forall f \in \mathcal{A}=C^{\infty}(M)
$$

et la déformation (47) de $F$ est un cas particulier de la :

PROPOSITION 3.- Soient $\mathcal{A}$ une algèbre involutive d'opérateurs dans $\mathcal{H}$ et $N=\mathcal{A}^{\prime}=\{T \in \mathcal{L}(\mathcal{H}) ; T a=a T \quad \forall a \in \mathcal{A}\}$ l'algèbre de von Neumann commutant de $\mathcal{A}$. 
a) L'égalité suivante définit une action du groupe $G=G L_{1}(N)$ des éléments inversibles de $N$ sur les opérateurs $F, F=F^{*}, F^{2}=1$

$$
g(F)=(\alpha F+\beta)(\beta F+\alpha)^{-1} \quad \forall g \in G
$$

où $\alpha=\frac{1}{2}\left(g-\left(g^{-1}\right)^{*}\right), \beta=\frac{1}{2}\left(g+\left(g^{-1}\right)^{*}\right)$.

b) On $a[g(F), a]=Y[F, a] Y^{*} \quad \forall a \in \mathcal{A}$, où $Y=(\beta F+\alpha)^{*-1}$.

L'égalité b) montre que pour tout idéal bilatère $J \subset \mathcal{L}(\mathcal{H})$ la condition

$$
[F, a] \in J
$$

est préservée par la déformation $F \rightarrow g(F)$. Comme seule la mesurabilité de la différentielle de Beltrami $\mu$ est requise pour que $m$ vérifie (49), seule la mesurabilité de la structure conforme sur $M$ est requise pour que le calcul quantique associé soit défini. De plus b) montre que la condition de régularité sur $a \in L^{\infty}(M)$ définie par (50) ne dépend que de la structure quasiconforme de la variété $M$ ([CST]). Un homéomorphisme local $\varphi$ de $\mathbb{R}^{n}$ est quasiconforme ssi il existe $K<\infty$ tel que

$$
H_{\varphi}(x)=\operatorname{Limsup}_{r \rightarrow 0} \frac{\max |\varphi(x)-\varphi(y)| ;|x-y|=r}{\min |\varphi(x)-\varphi(y)| ;|x-y|=r} \leq K, \forall x \in \text { Domaine } \varphi .
$$

Une structure quasiconforme sur une variété topologique $M_{n}$ est donnée par un atlas quasiconforme. La discussion ci-dessus s'applique au cas général ( $n$ pair) ([CST]) et montre que le calcul quantique est bien défini pour toute variété quasiconforme. Le résultat de D. Sullivan $[\mathrm{S}]$ basé sur [Ki] montre que toute variété topologique $M_{n}$, $n \neq 4$ admet une structure quasiconforme. En utilisant le calcul quantique et la cohomologie cyclique à la place du calcul différentiel et de la théorie de Chern-Weil, on obtient ([CST]) une formule locale pour les classes de Pontrjagin topologiques de $M_{n}$

\section{LA FORMULE DE L'INDICE LOCALE ET LA CLASSE FONDA- MENTALE TRANSVERSE}

Nous montrons dans cette section que le calcul infinitésimal ci-dessus permet le passage du local au global dans le cadre général des triplets spectraux $(\mathcal{A}, \mathcal{H}, D)$. Nous appliquons ensuite le résultat général au produit croisé d'une variété par le groupe des difféomorphismes. 
Nous ferons l'hypothèse de régularité suivante $\operatorname{sur}(\mathcal{A}, \mathcal{H}, D)$

$$
a \text { et }[D, a] \in \cap \operatorname{Dom} \delta^{k}, \forall a \in \mathcal{A}
$$

où $\delta$ est la dérivation $\delta(T)=[|D|, T]$.

Nous désignerons par $\mathcal{B}$ l'algèbre engendrée par les $\delta^{k}(a), \delta^{k}([D, a])$. La dimension d'un triplet spectral est bornée supérieurement par $p>0$ ssi $a(D+i)^{-1}$ est un infinitésimal d'ordre $\frac{1}{p}$ pour tout $a \in \mathcal{A}$. Quand $\mathcal{A}$ est unifère cela ne dépend que du spectre de $D$.

La notion précise de dimension est définie comme le sous-ensemble $\Sigma \subset \mathbb{C}$ des singularités des fonctions analytiques

$$
\zeta_{b}(z)=\operatorname{Trace}\left(b|D|^{-z}\right) \quad \operatorname{Re} z>p, b \in \mathcal{B}
$$

Nous supposerons que $\Sigma$ est discret et simple, i.e. que les $\zeta_{b}$ se prolongent à $\mathbb{C} / \Sigma$ avec des pôles simples en $\Sigma$.

Nous renvoyons à [CM2] pour le cas de spectre multiple.

L'indice de Fredholm de l'opérateur $D$ détermine une application additive, $K_{1}(\mathcal{A}) \stackrel{\varphi}{\rightarrow} \mathbb{Z}$ donnée par l'égalité

$$
\varphi([u])=\operatorname{Indice}(P u P), u \in G L_{1}(\mathcal{A})
$$

où $P$ est le projecteur $P=\frac{1+F}{2}, F=$ Signe $(D)$.

Cette application est calculée par l'accouplement entre $K_{1}(\mathcal{A})$ et la classe de cohomologie du cocycle cyclique suivant

$$
\tau\left(a^{0}, \ldots, a^{n}\right)=\operatorname{Trace}\left(a^{0}\left[F, a^{1}\right] \ldots\left[F, a^{n}\right]\right) \quad \forall a^{j} \in \mathcal{A}
$$

où $F=$ Signe $D$ et où $n$ est un entier impair $n \geq p$.

Le problème est que $\tau$ est difficile à déterminer en général car la formule (4) implique la trace ordinaire au lieu de la trace locale $f$.

Ce problème est résolu par la formule suivante :

THÉORÈME 4 ([CM2]).-Soit $(\mathcal{A}, \mathcal{H}, D)$ un triplet spectral vérifiant les hypothèses (1) et (2).

a) L'égalité $f P=\operatorname{Res}_{z=0}$ Trace $\left(P|D|^{-z}\right)$ définit une trace sur l'algèbre engendrée par $\mathcal{A},[D, \mathcal{A}]$ et $|D|^{z}, z \in \mathbb{C}$. 
b) La formule suivante n'a qu'un nombre fini de termes non nuls et définit les composantes $\left(\varphi_{n}\right)_{n=1,3, \ldots}$ d'un cocycle dans le bicomplexe $(b, B)$ de $\mathcal{A}$

$$
\varphi_{n}\left(a^{0}, \ldots, a^{n}\right)=\sum_{k} c_{n, k} f a^{0}\left[D, a^{1}\right]^{\left(k_{1}\right)} \ldots\left[D, a^{n}\right]^{\left(k_{n}\right)}|D|^{-n-2|k|} \quad \forall a^{j} \in \mathcal{A}
$$

où l'on note $T^{(k)}=\nabla^{k}(T)$ et $\nabla(T)=D^{2} T-T D^{2}$, et où $k$ est un multiindice, $c_{n, k}=(-1)^{|k|} \sqrt{2 i}\left(k_{1} ! \ldots k_{n} !\right)^{-1}\left(\left(k_{1}+1\right) \ldots\left(k_{1}+k_{2}+\ldots+k_{n}+n\right)\right)^{-1} \Gamma\left(|k|+\frac{n}{2}\right)$, $|k|=k_{1}+\ldots+k_{n}$.

c) L'accouplement de la classe, de cohomologie cyclique $\left(\varphi_{n}\right) \in H C^{*}(\mathcal{A})$ avec $K_{1}(\mathcal{A})$ donne l'indice de Fredholm de $D$ à coefficient dans $K_{1}(\mathcal{A})$.

Rappelons que le bicomplexe $(b, B)$ est donné par les opérateurs suivants agissant sur les formes multilinéaires sur l'algèbre $\mathcal{A}$

$$
\begin{gathered}
(b \varphi)\left(a^{0}, \ldots, a^{n+1}\right)= \\
\sum_{0}^{n}(-1)^{j} \varphi\left(a^{0}, \ldots, a^{j} a^{j+1}, \ldots, a^{n+1}\right)+(-1)^{n+1} \varphi\left(a^{n+1} a^{0}, a^{1}, \ldots, a^{n}\right) \\
B=A B_{0}, B_{0} \varphi\left(a^{0}, \ldots, a^{n-1}\right)=\varphi\left(1, a^{0}, \ldots, a^{n-1}\right)-(-1)^{n} \varphi\left(a^{0}, \ldots, a^{n-1}, 1\right) \\
(A \psi)\left(a^{0}, \ldots, a^{n-1}\right)=\sum_{0}^{n-1}(-1)^{(n-1) j} \psi\left(a^{j}, a^{j+1}, \ldots, a^{j-1}\right) .
\end{gathered}
$$

Nous renvoyons à $[\mathrm{Co}]$ pour la normalisation de l'accouplement entre $H C^{*}$ et $K(\mathcal{A})$.

Remarques. - a) L'énoncé du théorème 4 reste valable si l'on remplace dans toutes les formules l'opérateur $D$ par $D|D|^{\alpha}, \alpha \geq 0$.

b) Dans le cas pair, c'est-à-dire si l'on suppose que $\mathcal{H}$ est $\mathbb{Z} / 2$ gradué par $\gamma$, $\gamma=\gamma^{*}, \gamma^{2}=1, \gamma a=a \gamma \quad \forall a \in \mathcal{A}, \gamma D=-D \gamma$, on a une formule analogue pour un cocycle $\left(\varphi_{n}\right), n$ pair qui donne l'indice de Fredholm de $D$ à coefficient dans $K_{0}$. Cependant la composante $\varphi_{0}$ ne s'exprime pas en terme du résidu $f$ car elle est non locale pour $\mathcal{H}$ de dimension finie (cf. [CM2]).

c) Quand le spectre de dimension $\Sigma$ a de la multiplicité on a une formule analogue mais qui implique un nombre fini de termes correctifs, dont le nombre est borné indépendamment de la multiplicité (cf. [CM2]).

Le spectre de dimensions d'une variété $V$ est $\{0,1, \ldots, n\}, n=\operatorname{dim} V$, et est simple. La multiplicité apparaît pour les variétés singulières et les ensembles de Cantor donnent 
des exemples de points complexes, $z \notin \mathbb{R}$ dans ce spectre. Nous discutons maintenant une construction géométrique générale pour laquelle les hypothèses (1) et (2) sont vérifiées. Il s'agit de construire la classe fondamentale en $K$-homologie d'une variété $K$-orientée $M$ sans briser la symétrie du groupe $\operatorname{Diff}^{+}(M)$ des difféomorphismes de $M$ qui préservent la $K$-orientation. De manière plus précise nous cherchons un triplet spectral, $\left(C^{\infty}(M), \mathcal{H}, D\right)$ de la même classe de $K$-homologie que l'opérateur de Dirac associé à une métrique riemannienne (cf. I (11) et (12)) mais qui soit équivariant par rapport au groupe $\operatorname{Diff}^{+}(M)$ au sens de $[\mathrm{K}]$. Cela signifie que l'on a une représentation unitaire $\varphi \rightarrow U(\varphi)$ de $\operatorname{Diff}^{+}(M)$ dans $\mathcal{H}$ telle que

$$
U(\varphi) f U(\varphi)^{-1}=f \circ \varphi^{-1} \quad \forall f \in C^{\infty}(M), \varphi \in \operatorname{Diff}^{+}(M)
$$

et que

$$
U(\varphi) D U(\varphi)^{-1}-D \text { est borné pour tout } \varphi \in \operatorname{Diff}^{+}(M) \text {. }
$$

Lorsque $D$ est l'opérateur de Dirac associé à une structure riemannienne le symbole principal de $D$ détermine cette métrique et les seuls difféomorphismes qui vérifient (8) sont les isométries.

La solution de ce problème est essentielle pour définir la géométrie transverse des feuilletages et elle est effectuée en 2 étapes. La première est l'utilisation ([Co1]) de la métrique de courbure négative de l'espace $G L(n) / O(n)$ et de l'opérateur "dual Dirac" de Miscenko et Kasparov pour se ramener à l'action de $\operatorname{Diff}^{+}(M)$ sur l'espace total $P$ du fibré des métriques de $M$. La deuxième, dont l'idée est due à Hilsum et Skandalis ([HS]), est l'utilisation des opérateurs hypoelliptiques pour construire l'opérateur $D$ sur $P$.

On notera qu'alors que la géométrie équivariante obtenue pour $P$ est de dimension finie et vérifie les hypothèses (1) (2) la géométrie obtenue sur $M$ en utilisant le produit intersection avec le "dual Dirac" est de dimension infinie et $\theta$-sommable

$$
\operatorname{Trace}\left(e^{-\beta D^{2}}\right)<\infty \quad \forall \beta>0 .
$$

Par construction, le fibré $P \stackrel{\pi}{\rightarrow} M$ est le quotient $F / O(n)$ du $G L(n)$ fibré principal $F$ des repères sur $M$ par l'action du groupe orthogonal $O(n) \subset G L(n)$. L'espace $P$ admet la structure canonique suivante : le feuilletage vertical $V \subset T P, V=\operatorname{Ker} \pi_{*}$ et les structures euclidiennes suivantes sur les fibrés $V$ et $N=(T P) / V$. Le choix d'une métrique riemannienne $G L(n)$-invariante sur $G L(n) / O(n)$ détermine la métrique sur 


\section{A. CONNES}

$V$ et celle de $N$ est la métrique tautologique: $p \in P$ détermine une métrique sur $T_{\pi(p)}(M)$ qui grâce à $\pi_{*}$ est isomorphe à $N_{p}$.

Cette construction est fonctorielle pour les difféomorphismes de $M$.

Le calcul hypoelliptique adapté à cette structure est un cas particulier du calcul pseudodifférentiel sur les variétés de Heisenberg ([BG]). Il modifie simplement l'homogénéité des symboles $\sigma(p, \xi)$ en utilisant les homothéties :

$$
\lambda \cdot \xi=\left(\lambda \xi_{v}, \lambda^{2} \xi_{n}\right), \forall \lambda \in \mathbb{R}_{+}^{*}
$$

où $\xi_{v}, \xi_{n}$ sont les composantes verticales et normales du covecteur $\xi$. La formule (10) dépend de coordonnées locales $\left(x_{v}, x_{n}\right)$ adaptées au feuilletage vertical mais le calcul pseudodifférentiel correspondant n'en dépend pas. Le symbole principal d'un opérateur hypoelliptique d'ordre $k$ est une fonction, homogène de degré $k$ pour (10), sur le fibré $V^{*} \oplus N^{*}$. Le noyau distribution $k(x, y)$ d'un opérateur pseudodifférentiel $T$ dans le calcul hypoelliptique admet un développement au voisinage de la diagonale de la forme

$$
k(x, y) \sim \sum a_{j}(x, x-y)+a(x) \log |x-y|^{\prime}+0(1)
$$

où $a_{j}$ est homogène de degré $-j$ en $x-y$ pour (10) et où la métrique $|x-y|^{\prime}$ est localement de la forme

$$
|x-y|^{\prime}=\left(\left(x_{v}-y_{v}\right)^{4}+\left(x_{n}-y_{n}\right)^{2}\right)^{1 / 4} \text {. }
$$

Comme dans le calcul pseudodifférentiel ordinaire, le résidu se prolonge aux opérateurs de tout degré et est donné par l'égalité

$$
f T=\frac{1}{v+2 m} \int a(x)
$$

où la 1 -densité $a(x)$ ne dépend pas du choix de la métrique ||$^{\prime}$ et où $v=\operatorname{dim} V$, $m=\operatorname{dim} N$ de sorte que $v+2 m$ est la dimension de Hausdorff de l'espace métrique $\left(P,||^{\prime}\right)$.

L'opérateur $D$ est défini par l'équation $D|D|=Q$ où $Q$ est l'opérateur différentiel hypoelliptique de degré 2 obtenu en combinant (quand $v$ est pair) l'opérateur $d_{V} d_{V}^{*}$ $d_{V}^{*} d_{V}$ de signature où $d_{V}$ est la différentiation verticale, avec l'opérateur de Dirac transverse. (On utilise le revêtement métaplectique $M \ell(n)$ de $G L(n)$ pour définir la structure spinorielle sur $M$.) La formule explicite de $Q$ utilise une connection affine sur $M$ mais le choix de cette connection n'affecte pas le symbole principal hypoelliptique de 
$Q$ et donc de $D$ ce qui assure l'invariance (8) de $D$ par rapport aux difféomorphismes de $M$.

Donnons la formule explicite de $Q$ dans le cas $n=1$, i.e. pour $M=S^{1}$. On remplace $P$ par la suspension $S P=\mathbb{R} \times P$ pour se ramener au cas où la dimension verticale $v$ est paire. Un point de $S P=\mathbb{R} \times P$ est paramétré par 3 coordonnées $\alpha \in \mathbb{R}$ et $p=(s, \tau)$ où $\tau \in S^{1}$ et où $s \in \mathbb{R}$ définit la métrique $e^{2 s}(d \tau)^{2}$ en $\tau \in S^{1}$.

On munit $S P$ de la mesure $\nu=d \alpha d s d \tau$ et l'on représente l'algèbre $C_{c}^{\infty}(S P)$ par opérateurs de multiplication dans $\mathcal{H}=L^{2}(S P, \nu) \otimes \mathbb{C}^{2}$. La fonctorialité de la construction ci-dessus donne la représentation unitaire suivante du groupe $\operatorname{Diff}^{+}\left(S^{1}\right)$

$$
\left(U(\varphi)^{-1} \xi\right)(\alpha, s, \tau)=\varphi^{\prime}(\tau)^{1 / 2} \xi\left(\alpha, s-\log \varphi^{\prime}(\tau), \varphi(\tau)\right)
$$

Enfin l'opérateur $Q$ est donné par la formule

$$
Q=-2 \partial_{\alpha} \partial_{s} \sigma_{1}+\frac{1}{i} e^{-s} \partial_{\tau} \sigma_{2}+\left(\partial_{s}^{2}-\partial_{\alpha}^{2}-\frac{1}{4}\right) \sigma_{3}
$$

où $\sigma_{1}, \sigma_{2}, \sigma_{3} \in M_{2}(\mathbb{C})$ sont les 3 matrices de Pauli.

L'opérateur $\partial_{\tau}$ est de degré 2 dans le calcul hypoelliptique et l'on vérifie que $Q$ est hypoelliptique.

Un long calcul donne le résultat suivant ([CM3]) :

THÉORÈME 5. - Soit $\mathcal{A}$ l'algèbre produit croisé de $C_{c}^{\infty}(S P)$ par $\operatorname{Diff}^{+}\left(S^{\mathbf{1}}\right)$.

a) Le triplet spectral $(\mathcal{A}, \mathcal{H}, D)$ (où $\mathcal{A}$ agit dans $\mathcal{H}$ par (14) et $D|D|=Q$ ) satisfait les hypothèses (1) et (2) et son spectre de dimension est $\Sigma=\{0,1,2,3,4\}$.

b) La seule composante non nulle du cocycle associé (Théorème 4) est $\varphi_{3}$ et elle est cohomologue à $2 \psi$ où $\psi$ est le 3 -cocycle cyclique classe fondamentale transverse du produit croisé.

$L$ 'intégralité de $2 \psi$, i.e. de l'accouplement $\left\langle 2 \psi, K_{1}(\mathcal{A})\right\rangle$ résulte alors du théorème 4. Le 3-cocycle $\psi$ est donné par (cf. [Co])

$$
\psi\left(f^{0} U\left(\varphi_{0}\right), f^{1} U\left(\varphi_{1}\right), f^{2} U\left(\varphi_{2}\right), f^{3} U\left(\varphi_{3}\right)\right)=\int h^{0} d h^{1} \wedge d h^{2} \wedge d h^{3} \operatorname{si} \varphi_{0} \varphi_{1} \varphi_{2} \varphi_{3}=1
$$

$$
\begin{gathered}
\text { et }=0 \text { si } \varphi_{0} \varphi_{1} \varphi_{2} \varphi_{3} \neq 1 \\
\text { avec } h^{0}=f^{0}, h^{1}=\left(f^{1}\right)^{\varphi_{0}}, h^{2}=\left(f^{2}\right)^{\varphi_{0} \varphi_{1}}, h^{3}=\left(f^{3}\right)^{\varphi_{0} \varphi_{1} \varphi_{2}} .
\end{gathered}
$$

L'homologie entre $\varphi_{3}$ et $2 \psi$ met en évidence l'action sur l'algèbre $\mathcal{A}$ de l'algèbre de Hopf engendrée par les transformations linéaires suivantes (pour la relation de $\delta_{3}$ avec 


\section{A. CONNES}

l'invariant de Godbillon Vey, voir [Co]) de $\mathcal{A}$

$$
\begin{gathered}
\delta_{1}(f U(\varphi))=\left(\partial_{\alpha} f\right) U(\varphi), \delta_{2}(f U(\varphi))=\left(\partial_{s} f\right) U(\varphi) \\
\delta_{3}(f U(\varphi))=f e^{-s} \partial_{\tau} \log \left(\varphi^{-1}\right)^{\prime} U(\varphi), X(f U(\varphi))=e^{-s}\left(\partial_{\tau} f\right) U(\varphi)
\end{gathered}
$$

dont la compatibilité avec la multiplication de $\mathcal{A}$ est régie par le coproduit

$$
\Delta \delta_{j}=\delta_{j} \otimes 1+1 \otimes \delta_{j} \quad j=1,2,3
$$

(i.e. les $\delta_{j}$ sont des dérivations de $\mathcal{A}$ )

$$
\Delta X=X \otimes 1+1 \otimes X-\delta_{3} \otimes \delta_{2}
$$

où (19) montre que $X$ est de degré 2 .

\section{LA NOTION DE VARIÉTÉ ET LES AXIOMES DE LA GÉOMÉTRIE}

Commençons par spécifier la place de la géométrie riemannienne dans notre cadre en caractérisant (Théorème 6) les triplets spectraux correspondants. Soit $n \in \mathbb{N}$ la dimension, le triplet $(\mathcal{A}, \mathcal{H}, D)$ est supposé $\mathbb{Z} / 2$ gradué par $\gamma, \gamma=\gamma^{*}, \gamma^{2}=1$ quand $n$ est pair.

Les axiomes commutatifs sont les suivants :

1) (Dimension) $d s=D^{-1}$ est infinitésimal d'ordre $\frac{1}{n}$.

2) (Ordre un) $[[D, f], g]=0 \quad \forall f, g \in \mathcal{A}$.

3) (Régularité) Pour tout $f \in \mathcal{A}, f$ et $[D, f]$ appartiennent à $\underset{k}{\bigcap}$ Domaine $\delta^{k}$, où $\delta$ est la dérivation $\delta(T)=[|D|, T]$.

4) (Orientabilité) Il existe un cycle de Hochschild $c \in Z_{n}(\mathcal{A}, \mathcal{A})$ tel que $\pi(c)=1$ ( $n$ impair) ou $\pi(c)=\gamma$ ( $n$ pair), où $\pi: \mathcal{A}^{\otimes(n+1)} \rightarrow \mathcal{L}(\mathcal{H})$ est l'unique application linéaire telle que $\pi\left(a^{0} \otimes a^{1} \otimes \cdots \otimes a^{n}\right)=a^{0}\left[D, a^{1}\right] \ldots\left[D, a^{n}\right] \quad \forall a^{j} \in \mathcal{A}$.

5) (Finitude) Le $\mathcal{A}$-module $\mathcal{E}=\bigcap_{k}$ Domaine $D^{k}$ est projectif de type fini et l'égalité suivante définit une structure hermitienne sur $\mathcal{E}$

$$
\langle a \xi, \eta\rangle=f a(\xi, \eta) d s^{n} \quad \forall \xi, \eta \in \mathcal{E}, a \in \mathcal{A} .
$$

6) (Dualité de Poincaré) La forme d'intersection $K_{*}(\mathcal{A}) \times K_{*}(\mathcal{A}) \rightarrow \mathbb{Z}$ donnée par la composition de l'indice de Fredholm de $D$ avec la diagonale, $m_{*}: K_{*}(\mathcal{A}) \times K_{*}(\mathcal{A}) \rightarrow$ $K_{*}(\mathcal{A} \otimes \mathcal{A}) \rightarrow K_{*}(\mathcal{A})$, est inversible. 
7) (Réalité) Il existe une isométrie antilinéaire $J$ sur $\mathcal{H}$ telle que $J a^{*} J^{-1}=a \quad \forall a \in \mathcal{A}$ et $J^{2}=\varepsilon, J D=\varepsilon^{\prime} D J, J \gamma=\varepsilon^{\prime \prime} \gamma J$ où la table des valeurs de $\varepsilon, \varepsilon^{\prime}, \varepsilon^{\prime \prime} \in\{-1,1\}$ en fonction de $n$ modulo 8 est donnée en (I.16).

Les axiomes 2) et 4) donnent la présentation de l'algèbre abstraite notée $(\mathcal{A}, d s)$ engendrée par $\mathcal{A}$ et $d s=D^{-1}$.

THÉORÈME 6.- Soit $\mathcal{A}=C^{\infty}(M)$ où $M$ est une variété compacte de classe $C^{\infty}$.

a) Soit $\pi$ une représentation unitaire de $(\mathcal{A}, d s)$ satisfaisant les conditions 1$) \grave{a}$ 7). Il existe alors une unique structure riemannienne $g$ sur $M$ telle que la distance géodésique soit donnée par :

$$
d(x, y)=\operatorname{Sup}\{|a(x)-a(y)| ; a \in \mathcal{A},\|[D, a]\| \leq 1\} .
$$

b) La métrique $g=g(\pi)$ ne dépend que de la classe d'équivalence unitaire de $\pi$ et les fibres de l'application \{classe d'équivalence unitaire\} $\rightarrow g(\pi)$ forment un nombre fini d'espaces affines $\mathcal{A}_{\sigma}$ paramétrés par les structures spinorielles $\sigma$ de $M$.

c) La fonctionnelle $f d s^{n-2}$ est quadratique et positive sur chaque $\mathcal{A}_{\sigma}$ où elle admet un unique minimum $\pi_{\sigma}$.

d) $\pi_{\sigma}$ est la représentation de $(\mathcal{A}, d s)$ dans $L^{2}\left(M, S_{\sigma}\right)$ donnée par les opérateurs de multiplication et l'opérateur de Dirac associé à la connection de Levi Civita de la métrique $g$.

e) La valeur de $f d s^{n-2}$ en $\pi_{\sigma}$ est l'action de Hilbert Einstein de la métrique $g$ :

$$
f d s^{n-2}=-c_{n} \int r \sqrt{g} d^{n} x, c_{n}=\frac{n-2}{12}(4 \pi)^{-n / 2} 2^{[n / 2]} \Gamma\left(\frac{n}{2}+1\right)^{-1} .
$$

L'exemple le plus simple pour comprendre la signification du théorème est de vérifier que la géométrie du cercle $S^{1}$ de longueur $2 \pi$ est entièrement spécifiée par la présentation :

$$
U^{-1}[D, U]=1 \text {, où } U U^{*}=U^{*} U=1 .
$$

L'algèbre $\mathcal{A}$ étant celle des fonctions $C^{\infty}$ de l'opérateur unitaire $U$, on a $S^{1}=$ Spectre $(\mathcal{A})$ et l'égalité $(1)$ est le cas le plus simple de l'axiome 4 .

Remarques.- a) L'hypothèse $\mathcal{A}=C^{\infty}(M)$ devrait résulter des axiomes 1) - 7) (et de la commutativité de $\mathcal{A}$ ). Il résulte de 3 ) et 5) que si $\mathcal{A}^{\prime \prime}$ est l'algèbre de von Neumann engendrée par $\mathcal{A}$ on a :

$$
\mathcal{A}=\left\{T \in \mathcal{A}^{\prime \prime} ; T \in \bigcap_{k>0} \operatorname{Dom} \delta^{k}\right\},
$$


ce qui montre que $\mathcal{A}$ est uniquement spécifiée dans $\mathcal{A}^{\prime \prime}$ par la donnée de $D$. Cela montre que $\mathcal{A}$ est stable par calcul fonctionnel $C^{\infty}$ dans sa fermeture normique $A=\overline{\mathcal{A}}$ et en particulier que

$$
\text { Spectre } \mathcal{A}=\text { Spectre } A \text {. }
$$

Soit $X$ cet espace compact ; on devrait déduire des axiomes que l'application de $X$ dans $\mathbb{R}^{N}$ donnée par les $a_{i}^{j} \in \mathcal{A}$ qui interviennent dans le cycle de Hochschild $c$ de (4) est un plongement de $X$ comme sous-variété $C^{\infty}$ de $\mathbb{R}^{N}$ (cf. Proposition 15, p. 312 de $[\mathrm{Co}])$.

b) Rappelons qu'un cycle de Hochschild $c \in Z_{n}(\mathcal{A}, \mathcal{A})$ est un élément de $\mathcal{A}^{\otimes(n+1)}$, $c=\sum a_{i}^{0} \otimes a_{i}^{1} \ldots \otimes a_{i}^{n}$ tel que $b c=0$, où $b$ est l'application linéaire $b: \mathcal{A}^{\otimes n+1} \rightarrow \mathcal{A}^{\otimes n}$ telle que :

$$
\begin{gathered}
b\left(a^{0} \otimes \ldots \otimes a^{n}\right)= \\
\sum_{0}^{n-1}(-1)^{j} a^{0} \otimes \ldots \otimes a^{j} a^{j+1} \otimes \ldots \otimes a^{n}+(-1)^{n} a^{n} a^{0} \otimes a^{1} \otimes \ldots \otimes a^{n+1} .
\end{gathered}
$$

La classe de Hochschild du cycle $c$ détermine la forme volume.

c) Nous utilisons la convention selon laquelle la courbure scalaire $r$ est positive pour la sphère $S^{n}$, en particulier le signe de l'action $f d s^{n-2}$ est le bon pour la formulation euclidienne de la gravitation. Par exemple, pour $n=4$, l'action de Hilbert Einstein $-\frac{1}{16 \pi G} \int r \sqrt{g} d^{4} x$ coïncide avec l'aire $\frac{1}{\ell_{p}^{2}} f d s^{2}$ en unité de Planck.

d) Quand $M$ est une variété spinorielle l'application $\pi \rightarrow g(\pi)$ du théorème est surjective et si l'on fixe le cycle $c \in Z_{n}(\mathcal{A}, \mathcal{A})$ son image est l'ensemble des métriques dont la forme volume est fixée - (b)).

e) Si l'on supprime l'axiome 7 on a un résultat analogue au théorème en remplaçant les structures spinorielles par les structures $\operatorname{spin}^{c}([\mathrm{LM}])$, mais l'on n'a plus unicité dans c) à cause de la liberté dans le choix de la connection spinorielle.

f) Il résulte de l'axiome 4 et de ([Co], théorème $8, \mathrm{p} .309)$ que les opérateurs $a d s^{n}$, $a \in \mathcal{A}$ sont automatiquement mesurables de sorte que le symbole $f$ qui apparaît dans 5 est bien défini.

Passons au cas général non commutatif. Étant donnée une algèbre involutive $\mathcal{A}$ d'opérateurs dans l'espace de Hilbert $\mathcal{H}$, la théorie de Tomita [Ta] associe à tout vecteur $\xi \in \mathcal{H}$ cyclique pour $\mathcal{A}$ et pour son commutant $\mathcal{A}^{\prime}$

$$
\overline{\mathcal{A} \xi}=\mathcal{H}, \overline{\mathcal{A}^{\prime} \xi}=\mathcal{H}
$$


une involution antilinéaire isométrique $J: \mathcal{H} \rightarrow \mathcal{H}$ obtenue à partir de la décomposition polaire de l'opérateur

$$
S a \xi=a^{*} \xi \quad \forall a \in \mathcal{A}
$$

et qui vérifie la propriété de commutativité suivante :

$$
J \mathcal{A}^{\prime \prime} J^{-1}=\mathcal{A}^{\prime}
$$

On a donc en particulier $\left[a, b^{0}\right]=0 \quad \forall a, b \in \mathcal{A}$ où

$$
b^{0}=J b^{*} J^{-1} \quad \forall b \in \mathcal{A}
$$

de sorte que $\mathcal{H}$ devient un $\mathcal{A}$-bimodule en utilisant la représentation de l'algèbre opposée $\mathcal{A}^{0}$ donnée par (7). Dans le cas commutatif on a $a^{0}=a \quad \forall a \in \mathcal{A}$ de sorte que l'on ne perçoit pas la nuance entre module et bimodule.

Le théorème de Tomita est l'outil nécessaire pour assurer la substance des axiomes dans le cas général. Les axiomes 1) 3) et 5) sont inchangés, dans l'axiome de réalité 7) on remplace l'égalité $J a^{*} J^{-1}=a \quad \forall a \in \mathcal{A}$ par

$$
\left[a, b^{0}\right]=0 \quad \forall a, b \in \mathcal{A} \text { où } b^{0}=J b^{*} J^{-1}
$$

et l'axiome 2) (ordre un) se formule ainsi

$$
\left[[D, a], b^{0}\right]=0 \quad \forall a, b \in \mathcal{A}
$$

(On notera que comme $a$ et $b^{0}$ commutent $2^{\prime}$ équivaut à $\left[\left[D, a^{0}\right], b\right]=0 \quad \forall a, b \in \mathcal{A}$.) L'axiome $\left(7^{\prime}\right)$ fait de $\mathcal{H}$ un $\mathcal{A}$-bimodule et donne une classe $\mu$ de $K R^{n}$-homologie pour l'algèbre $\mathcal{A} \otimes \mathcal{A}^{0}$ munie de l'automorphisme antilinéaire $\tau$

$$
\tau\left(x \otimes y^{0}\right)=y^{*} \otimes x^{* 0} .
$$

Le produit intersection de Kasparov $[\mathrm{K}]$ permet alors de formuler la dualité de Poincaré, comme l'invertibilité de $\mu$

$$
\exists \beta \in K R_{n}\left(\mathcal{A}^{0} \otimes \mathcal{A}\right), \beta \otimes_{\mathcal{A}} \mu=\operatorname{id}_{\mathcal{A}^{0}}, \mu \otimes_{\mathcal{A}^{0}} \beta=\mathrm{id}_{\mathcal{A}} .
$$

Ceci implique l'isomorphisme $K_{*}(\mathcal{A}) \stackrel{\cap \mu}{\longrightarrow} K^{*}(\mathcal{A})$. La forme d'intersection

$$
K_{*}(\mathcal{A}) \times K_{*}(\mathcal{A}) \rightarrow \mathbb{Z}
$$




\section{A. CONNES}

est obtenue à partir de l'indice de Fredholm de $D$ à coefficient dans $K_{*}\left(\mathcal{A} \otimes \mathcal{A}^{0}\right)$ et n'utilise plus l'application diagonale $m: \mathcal{A} \otimes \mathcal{A} \rightarrow \mathcal{A}$ qui n'est un homomorphisme que dans le cas commutatif. Cette forme d'intersection est quadratique ou symplectique selon la valeur de $n$ modulo 8 .

L'homologie de Hochschild à coefficient dans un bimodule garde tout son sens dans le cas général et l'axiome 4) prend la forme suivante

$\left(4^{\prime}\right)$ Il existe un cycle de Hochschild $c \in Z_{n}\left(\mathcal{A}, \mathcal{A} \otimes \mathcal{A}^{0}\right)$ tel que $\pi(c)=1$ (n impair) ou $\pi(c)=\gamma(n$ pair $)$.

(Où $\mathcal{A} \otimes \mathcal{A}^{0}$ est le $\mathcal{A}$ bimodule obtenu par restriction à la sous-algèbre $\mathcal{A} \otimes 1 \subset \mathcal{A} \otimes \mathcal{A}^{0}$ de la structure de $\mathcal{A} \otimes \mathcal{A}^{0}$ bimodule de $\mathcal{A} \otimes \mathcal{A}^{0}$, i.e.

$$
\left.a\left(b \otimes c^{0}\right) d=a b d \otimes c^{0} \quad \forall a, b, c, d \in \mathcal{A} .\right)
$$

Les axiomes (1), (3) et (5) sont inchangés dans le cas noncommutatif et la démonstration de la mesurabilité des opérateurs $a(d s)^{n}, a \in \mathcal{A}$ reste valable en général. Nous adopterons les axiomes $(1),\left(2^{\prime}\right),(3),\left(4^{\prime}\right),(5),\left(6^{\prime}\right)$ et $\left(7^{\prime}\right)$ dans le cas général comme définition d'une variété spectrale de dimension $n$. L'algèbre $\mathcal{A}$ étant fixée nous parlerons de géométrie spectrale sur $\mathcal{A}$ comme dans I.20 et I.21. On démontre que l'algèbre de von Neumann $\mathcal{A}^{\prime \prime}$ engendrée par $\mathcal{A}$ dans $\mathcal{H}$ est automatiquement finie et hyperfinie et on a la liste complète de ces algèbres à isomorphisme près [Co]. L'algèbre $\mathcal{A}$ est stable par calcul fonctionnel $C^{\infty}$ dans sa fermeture normique $A=\overline{\mathcal{A}}$ de sorte que $K_{j}(\mathcal{A}) \simeq K_{j}(A)$, i.e. $K_{j}(\mathcal{A})$ ne dépend que de la topologie sous-jacente (définie par la $C^{*}$ algèbre $A$ ). L'entier $\chi=\langle\mu, \beta\rangle \in \mathbb{Z}$ donne la caractéristique d'Euler sous la forme

$$
\chi=\operatorname{Rang} K_{0}(\mathcal{A})-\operatorname{Rang} K_{1}(\mathcal{A})
$$

et le théorème 4 en donne une formule locale.

Le groupe $\operatorname{Aut}(\mathcal{A})$ des automorphismes de l'algèbre involutive $\mathcal{A}$ joue en général le rôle du groupe $\operatorname{Diff}(M)$ des difféomorphismes d'une variété $M$. (On a un isomorphisme canonique $\operatorname{Diff}(M) \stackrel{\alpha}{\rightarrow} \operatorname{Aut}\left(C^{\infty}(M)\right)$ donné par

$$
\left.\alpha_{\varphi}(f)=f \circ \varphi^{-1} \quad \forall f \in C^{\infty}(M), \varphi \in \operatorname{Diff}(M) .\right)
$$

Dans lè cas général non commutatif, parallèlement au sous-groupe normal $\operatorname{Int} \mathcal{A} \subset$ Aut $\mathcal{A}$ des automorphismes intérieurs de $\mathcal{A}$

$$
\alpha(f)=u f u^{*} \quad \forall f \in \mathcal{A}
$$


où $u$ est un élément unitaire de $\mathcal{A}$ (i.e. $u u^{*}=u^{*} u=1$ ), il existe un feuilletage naturel de l'espace des géométries spectrales sur $\mathcal{A}$ en classes d'équivalences formées des déformations intérieures d'une géométrie donnée. Une telle déformation est obtenue sans modifier ni la représentation de $\mathcal{A}$ dans $\mathcal{H}$ ni l'isométrie antilinéaire $J$ par la formule

$$
D \rightarrow D+A+J A J^{-1}
$$

où $A=A^{*}$ est un opérateur autoadjoint arbitraire de la forme

$$
A=\Sigma a_{i}\left[D, b_{i}\right], a_{i}, b_{i} \in \mathcal{A}
$$

Le nouveau triplet spectral obtenu continue à vérifier les axiomes $(1)-\left(7^{\prime}\right)$.

L'action du groupe $\operatorname{Int}(\mathcal{A})$ sur les géométries spectrales (cf. I.21) se réduit à une transformation de jauge sur $A$, donnée par la formule

$$
\gamma_{u}(A)=u\left[D, u^{*}\right]+u A u^{*}
$$

L'équivalence unitaire est implémentée par la représentation suivante du groupe unitaire de $\mathcal{A}$ dans $\mathcal{H}$

$$
u \rightarrow u J u J^{-1}=u\left(u^{*}\right)^{0} .
$$

La transformation (9) se réduit à l'identité dans le cas riemannien usuel. Pour obtenir un exemple non trivial, il suffit d'en faire le produit par l'unique géométrie spectrale sur l'algèbre de dimension finie $\mathcal{A}_{F}=M_{N}(\mathbb{C})$ des matrices $N \times N$ sur $\mathbb{C}, N \geq 2$. On a alors $\mathcal{A}=C^{\infty}(M) \otimes \mathcal{A}_{F}, \operatorname{Int}(\mathcal{A})=C^{\infty}(M, P S U(N))$ et les déformations intérieures de la géométrie sont paramétrées par les potentiels de jauge pour une théorie de jauge de groupe $S U(N)$. L'espace $P(\mathcal{A})$ des états purs de l'algèbre $\mathcal{A}$ est le produit $P=M \times P_{N-1}(\mathbb{C})$ et la métrique sur $P(\mathcal{A})$ déterminée par la formule $\mathrm{I} .10$ dépend du potentiel de jauge $A$. Elle coïncide avec la métrique de Carnot [G] sur $P$ définie par la distribution horizontale de la connection associée à $A$ (cf. [Co3]). Le groupe $\operatorname{Aut}(\mathcal{A})$ des automorphismes de $\mathcal{A}$ est le produit semi direct

$$
\operatorname{Aut}(\mathcal{A})=\mathcal{U} \rtimes \operatorname{Diff}(M)
$$

du groupe $\operatorname{Int}(\mathcal{A})$ des transformations de jauges locales par le groupe des difféomorphismes. En dimension $n=4$, les fonctionnelles d'action de Hilbert Einstein pour la métrique riemannienne et de Yang-Mills pour le potentiel vecteur $A$ apparaissent 
simplement, et avec les bons signes, dans le développement asymptotique en $\frac{1}{\Lambda} \mathrm{du}$ nombre $N(\Lambda)$ de valeurs propres de $D$ qui sont $\leq \Lambda$. On régularise cette expression en la remplaçant par

$$
\text { Trace } \varpi\left(\frac{D}{\Lambda}\right)
$$

où $\varpi \in C_{c}^{\infty}(\mathbb{R})$ est une fonction paire qui vaut 1 sur l'intervalle $[-1,1]$, (cf. [CC]). Les seuls autres termes non nuls du développement asymptotique sont un terme cosmologique, un terme de gravité de Weyl et un terme topologique.

Un exemple plus élaboré de variété spectrale est le tore non commutatif $\mathbb{T}_{\theta}^{2}$. Le paramètre $\theta \in \mathbb{R} / \mathbb{Z}$ définit la déformation suivante de l'algèbre des fonctions $C^{\infty}$ sur le tore $\mathbb{T}^{2}$, de générateurs $U, V$. Les relations

$$
V U=\exp 2 \pi i \theta U V \quad \text { et } \quad U U^{*}=U^{*} U=1, V V^{*}=V^{*} V=1
$$

définissent la structure d'algèbre involutive de $\mathcal{A}_{\theta}=\left\{\Sigma a_{n, m} U^{n} V^{n} ; a=\left(a_{n, m}\right) \in\right.$ $\left.\mathcal{S}\left(\mathbb{Z}^{2}\right)\right\}$ où $S\left(\mathbb{Z}^{2}\right)$ est l'espace de $S$ chwartz des suites à décroissance rapide. Comme pour les courbes elliptiques on utilise comme paramètre pour définir la géométrie de $\mathbb{T}_{\theta}^{2}$ un nombre complexe $\tau$ de partie imaginaire positive et, à isométrie près, cette géométrie ne dépend que de l'orbite de $\tau$ pour $\operatorname{PSL}(2, \mathbb{Z})[\mathrm{Co}]$. Le phénomène nouveau qui apparaît est l'équivalence de Morita qui relie entre elles les algèbres $\mathcal{A}_{\theta_{1}}, \mathcal{A}_{\theta_{2}}$ lorsque $\theta_{1}$ et $\theta_{2}$ sont dans la même orbite de l'action de $P S L(2, \mathbb{Z})$ sur $\mathbb{R}[\mathrm{Ri}]$.

Étant données une variété spectrale $(\mathcal{A}, \mathcal{H}, D)$ et une équivalence de Morita entre $\mathcal{A}$ et une algèbre $\mathcal{B}$ donnée par

$$
\mathcal{B}=\operatorname{End}_{\mathcal{A}}(\mathcal{E})
$$

où $\mathcal{E}$ est une $\mathcal{A}$-module à droite, projectif de type fini et hermitien, on obtient une géométrie spectrale sur $\mathcal{B}$ par le choix d'une connection hermitienne sur $\mathcal{E}$. Une telle connection $\nabla$ est une application linéaire $\nabla: \mathcal{E} \otimes_{\mathcal{A}} \Omega_{D}^{1}$ vérifiant les règles ([Co])

$$
\begin{gathered}
\nabla(\xi a)=(\nabla \xi) a+\xi \otimes d a \quad \forall \xi \in \mathcal{E}, a \in \mathcal{A} \\
(\xi, \nabla \eta)-(\nabla \xi, \eta)=d(\xi, \eta) \quad \forall \xi, \eta \in \mathcal{E}
\end{gathered}
$$

où $d a=[D, a]$ et où $\Omega_{D}^{1} \subset \mathcal{L}(\mathcal{H})$ est le $\mathcal{A}$-bimodule formé par les opérateurs de la forme (10). 
Toute algèbre $\mathcal{A}$ est Morita équivalente à elle-même (avec $\mathcal{E}=\mathcal{A}$ ) et quand on applique la construction ci-dessus on obtient les déformations intérieures de la géométrie spectrale.

\section{LA GÉOMÉTRIE SPECTRALE DE L'ESPACE TEMPS}

L'information expérimentale et théorique dont on dispose sur la structure de l'espace temps est résumée par la fonctionnelle d'action suivante $\mathcal{L}=\mathcal{L}_{E}+\mathcal{L}_{G}+$ $\mathcal{L}_{G \varphi}+\mathcal{L}_{\varphi}+\mathcal{L}_{\varphi f}+\mathcal{L}_{f}$, où $\mathcal{L}_{E}=-\frac{1}{16 \pi G} \int r \sqrt{g} d^{4} x$ est l'action de Hilbert-Einstein et les 5 autres termes constituent le modèle standard de la physique des particules, couplé de manière minimale à la gravitation. Outre la métrique $g_{\mu \nu}$ ce Lagrangien implique plusieurs champs de bosons et de fermions. Les bosons de spin 1 sont le photon $\gamma$, les bosons médiateurs $W^{ \pm}$et $Z$ et les huit gluons. Les bosons de spin 0 sont les champs de Higgs $\varphi$ qui sont introduits pour briser la parité et pour que le mécanisme de brisure de symétrie spontanée confère une masse aux diverses particules sans contredire la renormalisabilité des champs de jauge non abéliens. Tous les fermions sont de spin $\frac{1}{2}$ et forment 3 familles de quarks et leptons.

Les champs impliqués dans le modèle standard ont a priori un statut très différent de celui de la métrique $g_{\mu \nu}$. Le groupe de symétrie de ces champs, à savoir le groupe des transformations de jauge locales :

$$
\mathcal{U}=C^{\infty}(M, U(1) \times S U(2) \times S U(3))
$$

est a priori très différent du groupe $\operatorname{Diff}(M)$ de symétries de $\mathcal{L}_{E}$. Le groupe de symétrie naturel de $\mathcal{L}$ est le produit semidirect $\mathcal{U} \rtimes \operatorname{Diff}(M)=G$. La première question à résoudre si l'on veut donner une signification purement géométrique à $\mathcal{L}$ est de trouver un espace géométrique $X$ tel que $G=\operatorname{Diff}(X)$. Ceci détermine, en tenant compte du relèvement des difféomorphismes aux spineurs, l'algèbre $\mathcal{A}$ :

$$
\mathcal{A}=C^{\infty}(M) \otimes \mathcal{A}_{F}, \mathcal{A}_{F}=\mathbb{C} \oplus \mathbb{H} \oplus M_{3}(\mathbb{C})
$$

où l'algèbre involutive $\mathcal{A}_{F}$ est la somme directe des algèbres $\mathbb{C}, \mathbb{H}$ des quaternions et $M_{3}(\mathbb{C})$ des matrices $3 \times 3$ complexes.

L'algèbre $\mathcal{A}_{F}$ correspond à un espace fini dont les fermions du modèle standard et les paramètres de Yukawa (masses des fermions et matrice de mélange de Kobayashi Maskawa) déterminent la géométrie spectrale de la manière suivante. L'espace de 
Hilbert $\mathcal{H}_{F}$ est de dimension finie et admet pour base la liste des fermions élémentaires. Par exemple pour la 1ère génération de leptons cette liste est

$$
e_{L}, e_{R}, \nu_{L}, \bar{e}_{L}, \bar{e}_{R}, \bar{\nu}_{L}
$$

L'algèbre $\mathcal{A}_{F}$ admet une représentation naturelle dans $\mathcal{H}_{F}$ (cf. [Co3]) et en désignant par $J_{F}$ l'unique involution antilinéaire qui échange $f$ et $\bar{f}$ pour tout vecteur de la base, on a la commutation

$$
\left[a, J b^{*} J^{-1}\right]=0 \quad \forall a, b \in \mathcal{A}_{F} .
$$

L'opérateur $D_{F}$ est simplement donné par la matrice $\left[\begin{array}{cc}Y & 0 \\ 0 & \bar{Y}\end{array}\right]$ où $Y$ est la matrice de couplage de Yukawa. De plus les propriétés particulières de $Y$ assurent la commutation

$$
\left[\left[D_{F}, a\right], b^{0}\right]=0 \quad \forall a, b \in \mathcal{A}_{F} .
$$

La $\mathbb{Z} / 2$ graduation naturelle de $\mathcal{H}_{F}$ vaut 1 pour les fermions gauches $\left(e_{L}, \nu_{L} \ldots\right)$ et -1 pour les fermions droits ; on a

$$
\gamma_{F}=\varepsilon \varepsilon^{0} \text { où } \varepsilon=(1,-1,1) \in \mathcal{A}_{F} .
$$

Nous renvoyons à $[\mathrm{Co} 3]$ pour les vérifications des axiomes $(1)-\left(7^{\prime}\right)$. Le seul défaut est que le nombre de générations introduit une multiplicité dans la forme d'intersection, $K_{0}(\mathcal{A}) \times K_{0}(\mathcal{A}) \rightarrow \mathbb{Z}$, donnée par un multiple entier de la matrice $3 \times 3$

$$
\left[\begin{array}{ccc}
-1 & 1 & -1 \\
1 & 0 & 1 \\
-1 & 1 & 0
\end{array}\right] .
$$

Nous reviendrons à la fin de cet exposé sur la signification de la géométrie spectrale $\left(\mathcal{A}_{F}, \mathcal{H}_{F}, D_{F}\right)=F$.

Le pas suivant consiste à calculer les déformations intérieures (formule III.9) de la géométrie produit $M \times F$ où $M$ est une variété riemannienne spinorielle de dimension 4. Le calcul donne les bosons de jauge du modèle standard, $\gamma, W^{ \pm}, Z$, les huits gluons et les champs de Higgs $\varphi$ avec les bons nombres quantiques et montre que

$$
\mathcal{L}_{\varphi f}+\mathcal{L}_{f}=\langle\psi, D \psi\rangle
$$


où $D=D_{0}+A+J A J^{-1}$ est la déformation intérieure de la géométrie produit (donnée par l'opérateur $\left.D_{0}=\not \partial \otimes 1+\gamma_{5} \otimes D_{F}\right)$.

La structure de produit de $M \times F$ donne une bigraduation de $\Omega_{D}^{1}$ et une décomposition $A=A^{(1,0)}+A^{(0,1)}$ de $A$ qui correspond à la décomposition (8). Le terme $A^{(1,0)}$ rassemble tous les bosons de spin 1 et le terme $A^{(0,1)}$ les bosons de Higgs qui apparaissent comme des termes de différence finie sur l'espace $F$. Cette bigraduation existe sur l'analogue $\Omega_{D}^{2}$ des 2-formes ([Co]) et décompose la courbure $\theta=d A+A^{2}$ en trois termes $\theta=\theta^{(2,0)}+\theta^{(1,1)}+\theta^{(0,2)} 2$ à 2 orthogonaux pour le produit scalaire

$$
\left\langle\omega_{1}, \omega_{2}\right\rangle=f \omega_{1} \omega_{2}^{*} d s^{4} .
$$

Ainsi l'action de Yang-Mills, $\langle\theta, \theta\rangle=f \theta^{2} d s^{4}$ se décompose comme somme de 3 termes et on démontre que ces termes sont respectivement $\mathcal{L}_{G}, \mathcal{L}_{G \varphi}$ et $\mathcal{L}_{\varphi}$ pour $(2,0),(1,1)$ et $(0,2)$ respectivement [Co].

L'action de Yang-Mills $f \theta^{2} d s^{4}$ utilise la décomposition $D=D_{0}+A+J A J^{-1}$ et n'est donc pas, a priori, une fonction ne dépendant que de la géométrie définie par $D$. Nous avons vu en III.14 que, dans un cas plus simple, la combinaison $\mathcal{L}_{E}+\mathcal{L}_{G}$ apparaît directement dans le développement asymptotique du nombre de valeurs propres de $D$ inférieures à $\Lambda$. Le même principe (cf. [CC]) s'applique au modèle standard et conduit à la fonctionnelle suivante

$$
\text { Trace }\left(\varpi\left(\frac{D}{\Lambda}\right)\right)+\langle\psi, D \psi\rangle
$$

dont le développement asymptotique $([\mathrm{CC}])$ donne $\mathcal{L}+$ un terme de gravité de Weyl et un terme en $r \varphi^{2}$ qui est le seul terme que l'on peut rajouter à $\mathcal{L}$ sans altérer le modèle standard. Nous renvoyons à $[\mathrm{CC}]$ pour l'interprétation physique de ces résultats.

La géométrie finie $F$ ci-dessus était dictée par les résultats expérimentaux et il reste à en comprendre la signification conceptuelle à partir de l'analogue des groupes de Lie en géométrie non commutative, i.e. la théorie des groupes quantiques. Le fait simple (cf. $[\mathrm{M}]$ ) est que le revêtement spinoriel Spin(4) de $S O(4)$ n'est pas un revêtement maximal parmi les groupes quantiques. On a $\operatorname{Spin}(4)=S U(2) \times S U(2)$ et même le groupe $S U(2)$ admet grâce aux résultats de Lusztig des revêtements finis de la forme (Frobenius à l' $\infty$ ) :

$$
1 \rightarrow H \rightarrow S U(2)_{q} \rightarrow S U(2) \rightarrow 1,
$$

où $q$ est une racine de l'unité, $q^{m}=1, m$ impair. Le cas le plus simple est $m=3$, $q=\exp \left(\frac{2 \pi i}{3}\right)$. Le groupe quantique fini $H$ a une algèbre de Hopf de dimension 


\section{A. CONNES}

finie très voisine de $\mathcal{A}_{F}$, et la représentation spinorielle de $H$ définit un bimodule sur cette algèbre de Hopf de structure très voisine du bimodule $\mathcal{H}_{F}$ sur $\mathcal{A}_{F}$. Cela suggère d'étendre la géométrie spinorielle ([LM]) aux revêtements quantiques du groupe spinoriel, ce qui nécessite même pour parler de $G$-fibré principal, d'introduire un minimum de non commutativité (du style $C^{\infty}(M) \otimes \mathcal{A}_{F}$ ) dans l'algèbre des fonctions.

Mentionnons enfin que nous avons négligé dans cet exposé la nuance importante entre les signatures riemanniennes et lorentziennes.

\section{RÉFÉRENCES}

[At] M.F. Atiyah - K-theory and reality, Quart. J. Math. Oxford (2), 17 (1966), 367-386.

[B-G] R. Beals and P. Greiner - Calculus on Heisenberg manifolds, Annals of Math. Studies 119, Princeton Univ. Press, Princeton, N.J., 1988.

[B] T.P. Branson - An anomaly associated with 4-dimensional quantum gravity, to appear in C.M.P.

[B-O] T.P. Branson and B. Ørsted - Explicit functional determinants in four dimensions, Proc. Amer. Math. Soc. 113 (1991), 669-682.

[B-W] A.R. Bernstein and F. Wattenberg - Non standard measure theory, in Applications of model theory to algebra analysis and probability, Edited by W.A.J. Luxenburg Halt, Rinehart and Winstin (1969).

[C-J-G] A.H. Chamseddine, J. Fröhlich and O. Grandjean - The gravitational sector in the Connes-Lott formulation of the Standard model, J. Math. Phys. 36, $\mathrm{n}^{\circ} 11$ (1995).

[C-C] A. Chamseddine and A. Connes - The spectral action principle, to appear.

[Co] A. Connes - Noncommutative geometry, Academic Press (1994).

[Co1] A. Connes - Cyclic cohomology and the transverse fundamental class of a foliation, Geometric methods in operator algebras, (Kyoto, 1983), pp. 52-144, Pitman Res. Notes in Math. 123, Longman, Harlow (1986).

[Co2] A. Connes - Noncommutative geometry and reality, Journal of Math. Physics 36, $\mathrm{n}^{\circ} 11(1995)$.

[Co3] A. Connes - Gravity coupled with matter and the foundation of noncommutative geometry, to appear in C.M.P. 
[Co-L] A. Connes and J. Lott - Particle models and noncommutative geometry, Nuclear Phys. B. 18B (1990), suppl. 29-47 (1991).

[C-M1] A. Connes and H. Moscovici - Cyclic cohomology, the Novikov conjecture and hyperbolic groups, Topology 29 (1990), 345-388.

[C-M2] A. Connes and H. Moscovici - The local index formula in noncommutative geometry, GAFA 5 (1995), 174-243.

[C-M3] A. Connes and H. Moscovici - Hypoelliptic Dirac operator, diffeomorphisms and the transverse fundamental class.

[Co-S] A. Connes and G. Skandalis - The longitudinal index theorem for foliations, Publ. Res. Inst. Math. Sci. Kyoto 20 (1984), 1139-1183.

[C-S-T] A. Connes, D. Sullivan and N. Teleman - Quasiconformal mappings, operators on Hilbert space, and local formulae for characteristic classes, Topology, Vol. 33, $\mathrm{n}^{\circ} 4$ (1994), 663-681.

[D-T] T. Damour and J.H. Taylor - Strong field tests of relativistic gravity and binary pulsars, Physical Review D, Vol. 45, $\mathrm{n}^{\circ} 6$ (1992), 1840-1868.

[Dx] J. Dixmier - Existence de traces non normales, C.R. Acad. Sci. Paris, Ser. A-B 262 (1966).

[D-F-R] S. Doplicher, K. Fredenhagen and J.E. Roberts - Quantum structure of space time at the Planck scale and Quantum fields, to appear in CMP.

[F] J. Fröhlich - The noncommutative geometry of two dimensional supersymmetric conformal field theory, Preprint ETH (1994).

[G-F] K. Gawedzki and J. Fröhlich - Conformal Field theory and Geometry of Strings, CRM Proceedings and Lecture Notes, Vol. 7 (1994), 57-97.

[Gh] E. Ghys - L'invariant de Godbillon-Vey, Sém. Bourbaki 1988/89, exposé 706, Astérisque 177-178, S.M.F. (1989), 155-181.

[Gi] P. Gilkey - Invariance theory, the heat equation and the Atiyah-Singer index theorem, Math. Lecture Ser. 11, Publish or Perish, Wilmington, Del., 1984.

[G] M. Gromov - Carnot-Caratheodory spaces seen from within, Preprint IHES/ $\mathrm{M} / 94 / 6$.

[G-K-P] H. Grosse, C. Klimcik and P. Presnajder - On finite 4-dimensional quantum field theory in noncommutative geometry, CERN Preprint TH/96 - 51 Net Hepth/9602115.

[H-S] M. Hilsum, G. Skandalis - Morphismes K-orientés d'espaces de feuilles et fonctorialité en théorie de Kasparov, Ann. Sci. Ecole Norm. Sup. (4) 20 (1987), 325-390.

[I-K-S] B. Iochum, D. Kastler and T. Schücker - Fuzzy mass relations for the Higgs, J. 
Math. Phys. 36, $\mathrm{n}^{\circ} 11$ (1995).

[K-W] W. Kalau and M. Walze - Gravity, noncommutative geometry and the Wodzicki residue, J. of Geom. and Phys. 16 (1995), 327-344.

[K] G. Kasparov - The operator $K$-functor and extensions of $C^{*}$-algebras, Izv. Akad. Nauk. SSSR Ser. Mat. 44 (1980), 571-636.

[Ka] C. Kassel - Le résidu non commutatif, Sém. Bourbaki 1988/89, exposé 708, Astérisque 177-178, S.M.F. (1989), 199-229.

[Kas] D. Kastler - The Dirac operator and gravitation, Commun. Math. Phys. 166 (1995), 633-643.

[Ki] R.C. Kirby - Stable homeomorphisms and the annulus conjecture, Ann. Math. 89 (1969), 575-582.

[L-M] B. Lawson and M.L. Michelson - Spin Geometry, Princeton 1989.

[M] Y. Manin - Quantum groups and noncommutative geometry, Centre Recherche Math. Univ. Montréal (1988).

[Mi-S] J. Milnor and D. Stasheff - Characteristic classes, Ann. of Math. Stud. 76 Princeton University Press, Princeton, N.J. (1974).

[P] S. Power - Hankel operators on Hilbert space, Res. Notes in Math. 64, Pitman, Boston, Mass. (1982).

[Ri1] M.A. Rieffel - Morita equivalence for $C^{*}$-algebras and $W^{*}$-algebras, J. Pure Appl. Algebra 5 (1974), 51-96.

[Ri2] M.A. Rieffel - $C^{*}$-algebras associated with irrational rotations, Pacific J. Math. 93 (1981), 415-429; MR 83b:46087.

[R] B. Riemann - Mathematical Werke, Dover, New York (1953).

[St] E. Stein - Singular integrals and differentiability properties of functions, Princeton Univ. Press, Princeton, N.J. (1970).

[Ste] J. Stern - Le problème de la mesure, Sém. Bourbaki 1983/84, exposé 632, Astérisque 121-122 (1985), 325-346.

[S1] D. Sullivan - Hyperbolic geometry and homeomorphisms in Geometric Topology, Proceed. Georgia Topology Conf. Athens, Georgia (1977), 543-555.

[S2] D. Sullivan - Geometric periodicity and the invariants of manifolds, Lecture Notes in Math. 197, Springer (1971).

[Ta] M. Takesaki - Tomita's theory of modular Hilbert algebras and its applications, Lecture Notes in Math. 128, Springer (1970).

[W] Weinberg - Gravitation and Cosmology, John Wiley and Sons, New York London (1972). 
[Wo] M. Wodzicki - Noncommutative residue, Part I. Fundamentals K-theory, arithmetic and geometry, Lecture Notes in Math., 1289, Springer-Berlin (1987).

\author{
Alain CONNES \\ Collège de France \\ 3 , rue d'Ulm \\ 75005 PARIS \\ et \\ I.H.E.S. \\ 35 , route de Chartres \\ 91440 BURES-sur-YVETTE
}

